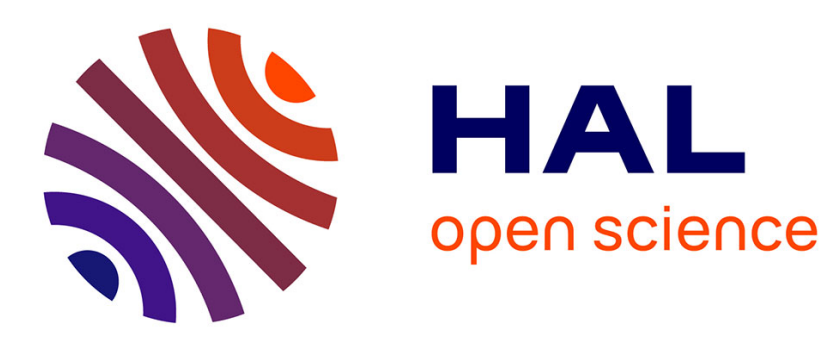

\title{
Theoretical principles of near-field optical microscopies and spectroscopies
}

\author{
Alain Dereux, Christian Girard, J. C. Weeber
}

\section{To cite this version:}

Alain Dereux, Christian Girard, J. C. Weeber. Theoretical principles of near-field optical microscopies and spectroscopies. Journal of Chemical Physics, 2000, 112 (18), pp.7775-7789. hal-00472372

\section{HAL Id: hal-00472372}

\section{https://hal.science/hal-00472372}

Submitted on 13 Apr 2010

HAL is a multi-disciplinary open access archive for the deposit and dissemination of scientific research documents, whether they are published or not. The documents may come from teaching and research institutions in France or abroad, or from public or private research centers.
L'archive ouverte pluridisciplinaire HAL, est destinée au dépôt et à la diffusion de documents scientifiques de niveau recherche, publiés ou non, émanant des établissements d'enseignement et de recherche français ou étrangers, des laboratoires publics ou privés. 


\title{
Theoretical principles of near-field optical microscopies and spectroscopies
}

\author{
Alain Dereux \\ Laboratoire de Physique de l'Université de Bourgogne, Optique Submicronique, \\ 9 avenue Alain Savary, F-21078 Dijon, France \\ Christian Girard \\ Centre d'Elaboration des Matériaux et d'Etudes Structurales, UPR CNRS 8011, \\ 29 rue Jeanne Marvig, F-31055 Toulouse, France \\ Jean-Claude Weeber \\ Laboratoire de Physique de l'Université de Bourgogne, Optique Submicronique, \\ 9 avenue Alain Savary, F-21078 Dijon, France
}

\begin{abstract}
This review deals with the principles of near-field optical signals detected near a surface in a manner permitting the mapping of the distribution of the fields close to various kinds of illuminated samples surfaces. We begin with a discussion of the main physical properties of the optical fields near a surface in the absence of any detector. This mainly concerns phenomena involving evanescent waves for which the local decay lengths are governed not only by the sizes but also by the intrinsic properties of the surface structures. The interpretation of the detection process in near-field optics is reviewed on the basis of a discussion about the possibility of establishing direct comparisons between experimental images and the solutions of Maxwell equations or the
\end{abstract}


electromagnetic local density of states.

\section{INTRODUCTION}

Over the last twenty years, a considerable amount of new developments were generated by taking advantage of different kinds of near-fields existing spontaneously or artificially close to the surfaces of various materials. In surface sciences, the practical use of these near-fields and of their local properties was latent for a long time until local probe based methods emerged at the beginning of the 1980s, namely: STM or Scanning tunneling Microscopy, AFM or Atomic Force Microscopy and finally NFO or Near-field Optics. This paper deals with the principles of NFO techniques which turn out to be a unique tool to map the distribution of optical fields close to various kinds of samples surfaces.

In surface physics, near-field optical phenomena were already actively studied $^{1-17}$ well before the locution "near-field optics" appeared in the mid 1980s with the first realizations of scanning near-field optical devices ${ }^{18^{-26}}$. Rapidly, the technical assets of the scanning near-field devices have offered new opportunities, i.e, detecting evanescent fields in guiding structures $^{27}$, imaging and exciting localized plasmons over a metallic surface ${ }^{28}$, mapping the luminescence generated at the surface of low-dimensional semiconductor structures ${ }^{29}$ or performing subwavelength near-field optical holography ${ }^{30}$. The reader can find in reference ${ }^{31}$ a detailed account of the early theoretical works on evanescent electromagnetic waves which play a central rôle in near-field optics. Instead of a historical review, the purpose of this paper is to focus on the crucial question of the interpretation of the detected signals by the various near-field optical devices.

\section{WHAT IS MEASURED BY A NEAR-FIELD OPTICAL MICROSCOPE?}

Until now, the search for techniques leading to optical resolution beyond the diffraction limit (to be refered below as "optical super-resolution") has somewhat occulted the basic question of the interpretation of the signal detected by near-field optical microscopes. 
Of course, one can expect that optical super-resolution should allow to observe smaller structures than those identified with a classical far-field optical microscope. However, the interpretation of experimental images mostly relied on the implicit intuitive assumption that the detected contrasts should allow to identify the exact shapes of the observed material structures. Psychologically, this assumption was so dominant that it led to a tendency to sort out the experimental results which met the expectation of perfect agreement between the optical signal and the exact shape of the underlying structures. However, as discussed in reference ${ }^{32}$, it turned out later that many of the cases where this agreement is found are suspected to be related to artefacts which may occur when controlling the tip motion with a feedback loop, not using the near-field optical signal, but using auxiliary AFM (shear-force) or STM signals. Moreover, although rarely mentionned clearly in the literature, it is an experimental fact that many (artefact free) recorded near-field optical images do not show this kind of agreement. Theoretical works ${ }^{33-35}$ intended then to recover the exact shapes of the observed material structures by applying mathematical techniques which are akin of inverse scattering and/or deconvolution methods. Such approaches still implicitly assume that near-field optical images should allow to identify the exact shapes of the underlying material structures, albeit after some data processing.

In this paper, we deliberately avoid to discuss about the principle of satisfying the expectation raised by this assumption. Instead, we propose a practical alternative to answer to the question of the interpretation of near-field optical images. For this purpose, we first summarize what kind of electromagnetic near-fields are known to exist in the vicinity of surfaces.

\section{A. Electromagnetic near-fields close to surfaces}

The surface limiting a solid body modifies locally the physical properties of many materials (dielectric, metal, or semi-conductor) ${ }^{36}$. Breaking the translational symmetry of homogeneous media leads to specific surface phenomena that have been well-identified for 
a long time (spontaneous polarization, electronic work function, electronic surface state, surface polaritons, surface enhanced optical properties, etc...).

In electrodynamics, a surface may be viewed as a place which can support different classes of near-fields. Actually, we have to distinguish two categories of surface electromagnetic near-fields:

(i) Surface electromagnetic near-fields which are the result of an external excitation (like photon or electron beams incident on a surface). They can be considered as the excited states of the photons field. Both optical near-fields and surface polaritons excited at a solid interface provide good illustrations of this category. Since they can be manipulated by an external operator, they are involved in the various optical near-field microscope setups ${ }^{26,37}$.

(ii) Quantum zero point fluctuations of electromagnetic fields which are confined near a solid-vacuum interface. Since they exist in the absence of any external excitation, one can view them as the ground state of the photons field. They are responsible for near-field dispersion effects, such as the van der Waals force field and the spontaneous interfacial polarization of the free surface of a dielectric. They thus play a significant rôle in local probe experiments such as $\mathrm{AFM}^{38,39}$.

\section{B. Probing electromagnetic near-fields locally}

At first sight, the main feature of all near-field optical microscopes is the nanometresized tip which is piezoelectrically driven to scan close to the sample surface. However, according to the experimental setup (see the review of existing devices in reference ${ }^{37}$ ), the tip may be used as a local photon emitter or as a local probe of the optical near-field. For reasons which will be made clear below, we will distinguish the various near-field optical microscope between passive probe devices and active probe devices. Among the passive probe devices, we find the PSTM (Photon Scanning Tunneling Microscope) ${ }^{22}$ or STOM 
(Scanning Tunneling Optical Microscope) ${ }^{23}$ and the family of so-called "apertureless" nearfield optical microscopes ${ }^{40-42}$ which all use their tips as local detectors only. PSTM exploit bare and sharply elongated optical fibres which may sometimes be coated with metals while apertureless microscopes are using bare metal tips.

The various transmission SNOM (Scanning Near-Field Optical Microscope) ${ }^{18,19}$ configurations belong to the category of active probe devices because their probe act as a local emitter of light. Reflection SNOM devices ${ }^{43}$ use their tips both as local emitter and local detector, but the discussion below will make clear that they are fundamentally active probe devices. SNOM favor metallized tips with a subwavelength aperture at the apex. Today, they are mostly obtained by coating a sharply elongated optical fibre. Recent alternatives involves tips with a tetrahedral termination (entirely or partly coated with metal) ${ }^{44}$ or microfabricated silicon-nitride tips with various shapes and coatings. In fact, as detailed in a recent review, the design of tips is still an open problem ${ }^{37}$. It mostly relies on empirical steps. The optimal tip characteristics for ensuring the best imaging properties are not well stated.

In the various theoretical descriptions of near-field optical devices, practical computational reasons require the simplification of the cumbersome task of taking the tip to sample coupling accurately into account. Cases where the interpretation of near-field optical images is problematic are often attributed to the approximations made in describing each kind of tip. Instead of discussing the principle of refining the description of this coupling, we propose here a practical point of view which is based on the distinct features of each family of probe devices.

The usual goal of a measurement is to provide informations about a physical system. Although quantum physics has shown that the situation may be complicated by entangled states, relevant informations are those describing some aspect of the physical system, aspect that is still supposed to exist in the absence of the measurement process. With the classification of section II A in mind, let us now consider what kinds of physical quantities do exist, close to a sample surface, at optical frequencies and in the absence of any measure- 
ment process, i.e. if no probe tip is present. In view of the above distinction, the physical quantities are of quite different natures according to the passive or active character of the probe devices. Let us begin by the simplest situation, namely the passive probe devices.

\section{Passive probe devices}

In this case, the illumination of the sample is not achieved through the tip but by a classical incident plane wave. In PSTM setups, the illuminating field is incident below the surface at an angle larger than the critical angle for total reflexion $\theta_{\text {tot }}$. Above a perfectly flat surface, the resulting incident field is a surface wave which decays exponentially along the $z$ direction (figure 1B).

Some apertureless microscopes also use the total internal reflection to excite surface plasmons of thin metal film ${ }^{40}$ : above the surface, the near-field of the surface plasmon also decays exponentially along the $z$ direction. Recently, apertureless microscopes were also used to study localized plasmons of random-metal dielectric films ${ }^{45}$. Other apertureless microscopes ${ }^{41,46}$ use the external illumination from the outside medium (figure 1A) so that the distribution of the electromagnetic near-field above the sample critically depends on the incident angle since, except at the Brewster angle, the field intensity is modulated by the interferences between incident and reflected waves.

For our practical purpose, we retain that the common feature of passive probe devices is that the electromagnetic near-fields which exist close to the sample surface when no tip is present belong to the category (i) of the classification of section II A. In the absence of the tip, the optical near-fields close to the sample surface correspond to excited state of the photons field. Theoretically, they are described by solving Maxwell equations where the incident field is defined by the experimental conditions. 


\section{Active probe devices}

In these devices, the incident field is due to the emitting tip which is assumed to deliver a spatially localized source of light ${ }^{47-51}$. The electromagnetic near-fields which exist close to the sample surface when no tip is present belong thus to the category (ii) of the classification of section II A. In the absence of the tip, the optical near-fields correspond to the ground state of the photons field, i.e. to the electromagnetic near-fields which exist spontaneously in the absence of any external driving excitation.

In the case of active probe devices, the proposed practical point of view brings to the fore that the quantity which could be expected to be measured is related to the local density of states (LDOS) of the electromagnetic field. Since it describes the distribution of the electromagnetic field when there is not any incident field, the LDOS is not a commonly used concept in optics textbooks. Probably, the best known exemple of the use of this concept occurs in Planck's description of the black body radiation where the LDOS multiplies the Bose-Einstein distribution. In this context, the formula of the LDOS is derived in a straightforward manner. However, in the context of near-field optics discussed here, obtaining a theoretical distribution of the LDOS turns out to be computationally more demanding.

\section{E. Interpretation of the images}

The following work program is required to check the validity of the hypothesis underlying the practical point of view developed in this section:

(a) Are the images obtained by passive probe devices in agreement with the distribution of the electromagnetic near-field scattered by the sample surface, as computed without including any tip?

(b) Is there any link between the images obtained by active probe devices and the distribution of the electromagnetic local density of states close to the sample surface, as 
computed without including any tip?

The section $\mathrm{V}$ below reviews recent results which support a positive answer to the point (a) of the above program. However, the status of point (b) should be understood as a suggestion aimed at finding an issue to the difficult problem of the interpretation of the images obtained with active probe devices.

\section{BASIC THEORY}

The practical point of view proposed in section II requires a single theoretical framework which enables, close to the sample surface, the computation of the scattering of an electromagnetic field in the case of passive probe devices and a description of the LDOS in the case of active probe devices. These both features are delivered by a formulation based on the use of Green dyadics, also called propagators or field susceptibilities which we summarize in this section ${ }^{52-55}$.

\section{A. Scattering theory}

With the usual $\exp (-i \omega t)$ time dependence, the vector wave equation issued from Maxwell's equations (CGS units):

$$
-\nabla \times \nabla \times \mathbf{E}(\mathbf{r}, \omega)+\frac{\omega^{2}}{c^{2}} \epsilon(\mathbf{r}, \omega) \mathbf{E}(\mathbf{r}, \omega)=0
$$

may be cast as

$$
-\nabla \times \nabla \times \mathbf{E}(\mathbf{r}, \omega)+q^{2} \mathbf{E}(\mathbf{r}, \omega)=\mathbf{V}(\mathbf{r}, \omega) \mathbf{E}(\mathbf{r}, \omega)
$$

with

$$
q^{2}=\frac{\omega^{2}}{c^{2}} \epsilon_{r e f}
$$

Any complicated behavior due the anisotropy or to the low-symmetry of the geometrical shape of the original dielectric tensor profile $\epsilon(\mathbf{r}, \omega)$ is described as a difference relatively to the reference system $\epsilon_{\text {ref }}$ ( $\mathbf{1}$ is the unit dyadic): 


$$
\chi(\mathbf{r}, \omega)=\frac{1}{4 \pi}\left(\epsilon(\mathbf{r}, \omega)-\mathbf{1} \epsilon_{r e f}\right)
$$

The implicit Lippmann-Schwinger equation provides a solution of equation (2):

$$
\begin{aligned}
\mathbf{E}(\mathbf{r}, \omega) & =\mathbf{E}_{0}(\mathbf{r}, \omega) \\
& +\int_{V} d \mathbf{r}^{\prime} \mathbf{S}_{0}\left(\mathbf{r}, \mathbf{r}^{\prime}, \omega\right) \cdot \chi\left(\mathbf{r}^{\prime}, \omega\right) \cdot \mathbf{E}\left(\mathbf{r}^{\prime}, \omega\right) .
\end{aligned}
$$

In scattering theory, the first term $\mathbf{E}_{0}(\mathbf{r}, \omega)$ is refered as the incident field while the second term is called the scattered field obtained from the integration over the domain $V$ where $\chi\left(\mathbf{r}^{\prime}, \omega\right)$ is non-zero. $V$ defines the volume of the scatterer relatively to the reference system. This last equation can easily be solved numerically ${ }^{31}$ so that it provides a useful tool for the evaluation of the theoretical near-field distributions which are needed to check the point (a) of section IIE.

To solve the Lippmann-Schwinger equation, we need to know the analytical solution $\mathbf{E}_{0}(\mathbf{r}, \omega)$ satisfying

$$
-\nabla \times \nabla \times \mathbf{E}_{0}(\mathbf{r}, \omega)+q^{2} \mathbf{E}_{0}(\mathbf{r}, \omega)=0
$$

and the associated Green's dyadic defined by

$$
\begin{aligned}
-\nabla \times \nabla \times \mathbf{S}_{0}\left(\mathbf{r}, \mathbf{r}^{\prime}, \omega\right) & +q^{2} \mathbf{S}_{0}\left(\mathbf{r}, \mathbf{r}^{\prime}, \omega\right) \\
& =-\frac{4 \pi c^{2}}{\omega^{2}} \mathbf{1} \delta\left(\mathbf{r}-\mathbf{r}^{\prime}\right)
\end{aligned}
$$

where $\delta\left(\mathbf{r}-\mathbf{r}^{\prime}\right)$ is the Dirac delta function. The reference structure $\epsilon_{r e f}$ is usually a homogeneous background material or a semi-infinite surface system so that $\mathbf{E}_{0}(\mathbf{r}, \omega)$ is known analytically. For homogeneous media, the analytical form of $\mathbf{S}_{0}\left(\mathbf{r}, \mathbf{r}^{\prime}, \omega\right)$ is known from ancient works ${ }^{56,57}$. For a surface system, the expression of the propagator is somewhat more elaborated but is also found in the literature ${ }^{1,17,58}$.

\section{B. Local density of states}

Well-known in solid state physics ${ }^{59}$, the use of the LDOS is not commonly spread in electrodynamics ${ }^{1,60}$. Solid state physics applies the concept of LDOS to non-relativistic 
electrons so that the LDOS corresponds to the density of probability to find an electron of energy $\hbar \omega$ at the point $\mathbf{r}$ of the solid. This function is directly related to the square moduli of all possible electronic wavefunctions associated to this energy. In the case of photons, different formulations of the LDOS can be proposed depending on the reference field. The most widely used formulation relies on the calculation of the electric field susceptibility The LDOS is then deduced from the electric Green's dyadic $\mathbf{S}\left(\mathbf{r}, \mathbf{r}^{\prime}, \omega\right)$ of a sytem

$$
\rho(\mathbf{r}, \omega)=\frac{c^{2}}{\pi \omega^{2}} \Im \text { Trace } \mathbf{S}(\mathbf{r}, \mathbf{r}, \omega)
$$

where $\Im$ denotes the imaginary part. The vector character of electromagnetic fields allows to view this LDOS as the sum

$$
\rho(\mathbf{r}, \omega)=\sum_{j=x, y, z} \rho_{j j}(\mathbf{r}, \omega)
$$

where the "partial" LDOS are defined by:

$$
\rho_{j j}(\mathbf{r}, \omega)=\frac{c^{2}}{\pi \omega^{2}} \Im \mathbf{S}_{j j}(\mathbf{r}, \mathbf{r}, \omega)
$$

With this reference field, the LDOS is related to the square moduli of the electric field associated to all electromagnetic eigenmodes of frequency $\omega$. When this quantity is defined on the basis of any kind of mixed field-susceptibilities, such straightforward relation to the electric field is not possible anymore. However, in any case, the LDOS is the only quantitative way to describe the continuous part of the spectrum of any system independently of the excitation mode. In the context of optics, this means that the LDOS provides spectroscopic informations which are intrinsically independent of any particular illumination mode. This independence is exactly the basic feature which is postulated in the practical point of view developped in section II.

Within the framework of the linear response theory, the propagator entering equation (8) provides the value of the electric field (electric or magnetic) at an observation point $\mathbf{r}$ and a point-like source located at $\mathbf{r}^{\prime}$. This propagator is a second rank tensor which verifies a dyadic Dyson equation: 


$$
\begin{aligned}
\mathbf{S}\left(\mathbf{r}, \mathbf{r}^{\prime}, \omega\right) & =\mathbf{S}_{0}\left(\mathbf{r}, \mathbf{r}^{\prime}, \omega\right) \\
& +\int_{V} \mathbf{S}_{0}\left(\mathbf{r}, \mathbf{r}^{\prime}, \omega\right) \cdot \chi\left(\mathbf{r}^{\prime}, \omega\right) \cdot \mathbf{S}\left(\mathbf{r}, \mathbf{r}^{\prime}, \omega\right) d \mathbf{r}^{\prime}
\end{aligned}
$$

so that it may be deduced using the propagator $\mathbf{S}_{0}\left(\mathbf{r}, \mathbf{r}^{\prime}, \omega\right)$ of a reference system (homogeneous medium or surface) that we defined above $e^{61-63}$.

\section{APPLICATION OF THE THEORY TO PASSIVE PROBE DEVICES}

This section presents a simple application of the above formalism in order to model the near-field distribution close to a particle deposited on a surface.

\section{A. Decay length of surface optical waves}

In the TIR configuration, the basic surface wave is generated by illuminating the surface through the substrate by a monochromatic planewave of frequency $\omega_{0}$, incident at an angle $\theta$ larger than $\theta_{\text {tot }}$. Two different incident polarizations can be considered: the $s$ polarization, where the incident electric field is parallel to the surface and the $p$ polarization, where the incident electric field is in the plane of incidence.

The incident field at an observation point $\mathbf{r}=(x, y, z)=(\mathbf{l}, z)$ above the surface ( see figure (1B)) reads

$$
\mathbf{E}_{\mathbf{0}}(\mathbf{r}, \omega)=\mathbf{E}_{\mathbf{0}} e^{i \mathbf{k} \cdot \mathbf{l}} e^{-z / \eta}
$$

where

$$
\eta^{-1}=\frac{\omega}{c}\left(\sin ^{2} \theta-\sin ^{2} \theta_{\text {tot }}\right)^{1 / 2}
$$

and

$$
\|\mathbf{k}\|=\frac{\omega_{0}}{c} \sin \theta
$$

When, for example, the incident field is propagating along the $y$-axis, one obtains for the $s$ polarization: 


$$
\begin{aligned}
& E_{0 x}(\mathbf{r})=A_{0} T_{s} \\
& E_{0 y}(\mathbf{r})=E_{0 z}(\mathbf{r})=0 ;
\end{aligned}
$$

and for the $p$ polarization:

$$
\begin{aligned}
& E_{0 x}(\mathbf{r})=0 \\
& E_{0 y}(\mathbf{r})=A_{0} T_{p} \delta_{c} \\
& E_{0 z}(\mathbf{r})=A_{0} T_{p} \delta_{s} ;
\end{aligned}
$$

where

$$
\begin{aligned}
& \delta_{s}=\frac{\sin (\theta)}{\sin \left(\theta_{t o t}\right)}, \\
& \delta_{c}=\frac{i \eta}{k_{0} \sin \left(\theta_{t o t}\right)} .
\end{aligned}
$$

In equations (15) and (16), $A_{0}$ is proportional to $e^{i k y} e^{-z / \eta}$, and the factors $T_{s}$ and $T_{p}$ are the usual transmission coefficients for each polarization ${ }^{64}$. Through Maxwell equations, the magnetic field $\mathbf{B}_{0}(\mathbf{r})$ associated with the surface wave is then deduced from the relations (12), (15) and (16):

$$
\mathbf{B}_{0}(\mathbf{r}, \omega)=\frac{c}{i \omega} \nabla \wedge \mathbf{E}_{0}(\mathbf{r}, \omega)
$$

These last four equations completely define the electromagnetic state of the surface optical wave. The decay length $\eta$ of the electromagnetic evanescent field is defined by analyzing either the variations of the electric field or of the magnetic field intensities as a function of the distance $z$ from the sample. For the perfect planar surface considered in this section, $\eta$ does not depend on the polarization state. It just depends on the contrast of index of refraction (implicitly contained in the parameter $\theta_{\text {tot }}$ through Snell's law) and, as described in figure (2), on the angle of incidence $\theta$. Near the grazing angle $\left(\theta \sim 90^{\circ}\right)$ this parameter takes large values and the optical evanescent wave vanishes. 


\section{B. Modelling the optical response of particle on a flat surface}

The presence of structures buried in the substrate, protrusions lying on the surface, or any other kind of surface defects modifies significantly the state of the surface optical wave described in the preceding section IV A. To illustrate the interaction between an optical evanescent wave and a subwavelength size object lying on a sample surface, we consider the model spherical system whose geometrical parameters are described in figure 3. For optical wavelengths, the subwavelength regime covers the range of nanoscopic sizes. In order to be as simple as possible, the optical response of the sphere is modelled by a point-like polarizability $\alpha_{0}(\omega)$ which will allow to tackle the problem analytically. Indeed, the susceptibility $\chi(\mathbf{r}, \omega)$ is reduced to:

$$
\chi(\mathbf{r}, \omega)=\alpha_{0}(\omega) \delta\left(\mathbf{r}-\mathbf{r}_{s}\right)
$$

where $\mathbf{r}_{s}=(0,0, D)$ points to the particle position.

The Lippmann-Schwinger equation (5) can then be handled algebraically since the pointlike nature of the model optical response avoids the complication due to the integration. This allows to bring to the fore ${ }^{17,65-67}$ how the substrate modifies the polarizability $\alpha_{0}(\omega)$ of the particle into the effective polarizability tensor $\alpha$ through:

$$
\alpha\left(\mathbf{r}_{s}, \omega\right)=\alpha_{0}(\omega) \cdot \mathbf{M}\left(\mathbf{r}_{s}, \omega\right)
$$

with

$$
\mathbf{M}\left(\mathbf{r}_{s}, \omega\right)=\left[\mathbf{1}-\mathbf{S}_{0}\left(\mathbf{r}_{s}, \mathbf{r}_{s}, \omega\right) \cdot \alpha_{0}(\omega)\right]^{-1}
$$

where $\mathbf{S}_{0}\left(\mathbf{r}_{s}, \mathbf{r}_{s}, \omega\right)$ is the nonretarded propagator associated with the bare surface. In the context of molecular physics, the sphere models a molecule and $\alpha$ is called the "dressed" polarizability which describes the optical properties of the spherical particle-surface system. In the past, theoretical works ${ }^{17}$ have been devoted to the calculation of the dressed polarizabilities of molecules interacting with various substrates (spheres, cylinders, planes, ...). If the polarizability $\alpha_{0}(\omega)$ of the isolated particle is isotropic, the symmetry of the tensor 
$\alpha\left(\mathbf{r}_{s}, \omega\right)$ is mainly determined by the symmetry of the substrate. In the specific case of a single spherical particle interacting with a planar surface, the dyadic tensor $\mathbf{M}\left(\mathbf{r}_{s}, \omega\right)$ turns out to be diagonal. Consequently, $\alpha\left(\mathbf{r}_{s}, \omega\right)$ belongs to the $C_{\infty v}$ symmetry group. In this case, the tensor $\alpha$ involoves only two independent scalar components $\alpha_{\|}$and $\alpha_{\perp}{ }^{17,31,67}$

$$
\alpha\left(\mathbf{r}_{s}, \omega\right)=\left(\begin{array}{ccc}
\alpha_{\|}\left(\mathbf{r}_{s}, \omega\right) & 0 & 0 \\
0 & \alpha_{\|}\left(\mathbf{r}_{s}, \omega\right) & 0 \\
0 & 0 & \alpha_{\perp}\left(\mathbf{r}_{s}, \omega\right)
\end{array}\right)
$$

with

$$
\alpha_{\|}\left(\mathbf{r}_{s}, \omega\right)=\frac{8 \alpha_{0}(\omega) D^{3}}{8 D^{3}-\alpha_{0}(\omega) \Delta(\omega)}
$$

and

$$
\alpha_{\perp}\left(\mathbf{r}_{s}, \omega\right)=\frac{4 \alpha_{0}(\omega) D^{3}}{4 D^{3}-\alpha_{0}(\omega) \Delta(\omega)}
$$

In these two relations, the factor $\Delta(\omega)=\frac{(\epsilon(\omega)-1)}{(\epsilon(\omega)+1)}$ is merely the nonretarded reflection coefficient of the surface. Let us notice that when the particle and the substrate are made of dielectric materials of low optical indexes, the anisotropic ratio defined by

$$
\xi=\frac{\alpha_{\perp}}{\alpha_{\|}}
$$

remains always close to unity over all the optical spectrum. In this case, the polarizability $\alpha_{0}(\omega)$ of the free particle provides an excellent approximation of the effective polarizability tensor $\alpha\left(\mathbf{r}_{s}, \omega\right)$. This approximation is no longer valid when dealing with metallic objects for which all further calculations must be performed on the basis of equations (23) and (24).

\section{Contrast in the near-field zone}

We now deal with the interaction of the model particle with an incident optical surface wave set up by total internal reflection as described in section IV A. We focus on the nearfield zone, defined here as the points $\mathbf{r}$ located above the sample close the particle. In this 
zone, the incident surface wave is locally distorted by the interaction with the particle. To keep the matter as simple as possible, we consider a particle which satisfies the criterion defined by equation (25) so that we can use the first Born approximation. In this case, we obtain analytically tractable formulas for both electric $\mathbf{E}(\mathbf{r}, \omega)$ and magnetic $\mathbf{B}(\mathbf{r}, \omega)$ fields scattered by the fluctuating dipole moment $\mu\left(\mathbf{r}_{s}, \omega\right)=\alpha\left(\mathbf{r}_{s}, \omega\right) \cdot \mathbf{E}_{0}\left(\mathbf{r}_{s}, \omega\right)$ induced inside the particle:

$$
\begin{aligned}
\mathbf{E}(\mathbf{r}, \omega) & =\mathbf{E}_{0}(\mathbf{r}, t) \\
& +\mathbf{S}_{0}\left(\mathbf{r}, \mathbf{r}_{s}, \omega\right) \cdot \alpha\left(\mathbf{r}_{s}, \omega\right) \cdot \mathbf{E}_{0}\left(\mathbf{r}_{s}, \omega\right)
\end{aligned}
$$

and

$$
\begin{aligned}
\mathbf{B}(\mathbf{r}, \omega) & =\mathbf{B}_{0}(\mathbf{r}, \omega) \\
& +\mathbf{Q}_{0}\left(\mathbf{r}, \mathbf{r}_{s}, \omega\right) \cdot \alpha\left(\mathbf{r}_{s}, \omega\right) \cdot \mathbf{E}_{0}\left(\mathbf{r}_{s}, \omega\right)
\end{aligned}
$$

Above the substrate, we now approximate the dyadic tensors $\mathbf{S}_{0}\left(\mathbf{r}, \mathbf{r}_{s}, \omega\right)$ and $\mathbf{Q}_{0}\left(\mathbf{r}, \mathbf{r}_{s}, \omega\right)$ by the free space propagators which describe the fields radiated by an arbitrarily oriented $\operatorname{dipole}^{68}$. In the near-field zone, i.e. when $\left|\mathbf{r}-\mathbf{r}_{s}\right|<2 \pi c / \omega$, neglecting retardation effects allows to write these propagators as:

$$
\begin{gathered}
\mathbf{S}_{0}\left(\mathbf{r}, \mathbf{r}_{s}, \omega\right)=\nabla_{\mathbf{r}} \nabla_{\mathbf{r}}\left\{\frac{1}{\left|\mathbf{r}-\mathbf{r}_{s}\right|}\right\} \\
\mathbf{Q}_{0}\left(\mathbf{r}, \mathbf{r}_{s}, \omega\right)=\frac{i \omega}{c\left|\mathbf{r}-\mathbf{r}_{s}\right|^{3}}\left(\begin{array}{ccc}
0 & -z+D & y \\
z-D & 0 & -x \\
-y & x & 0
\end{array}\right)
\end{gathered}
$$

Quite often, in the experimental images provided by passive probe devices, small surface protrusions may appear with either a dark or a bright contrast, corresponding to a smaller or a larger number of detected photons. From Equations (26) and (27), we are now able to deduce four useful analytical expressions bringing to the fore the explicit dependence of the near-field intensity constrast with respect to the external parameters. This is achieved by defining two dimensionless coefficients depending on the location of the observation point $\mathbf{r}$ : 


$$
\Xi_{e}(\mathbf{r})=\frac{|\mathbf{E}(\mathbf{r}, \omega)|^{2}}{\left|\mathbf{E}_{0}(\mathbf{r}, \omega)\right|^{2}}-1
$$

and

$$
\Xi_{m}(\mathbf{r})=\frac{|\mathbf{B}(\mathbf{r}, \omega)|^{2}}{\left|\mathbf{B}_{0}(\mathbf{r}, \omega)\right|^{2}}-1
$$

When the observation point (that could be physically materialized by a sharp probe) is located just on the top of the particle (i.e., when $\left.\mathbf{r}=\mathbf{R}_{0}=\left(0,0, Z_{0}\right)\right)$, both the sign and magnitude of these coefficients provide direct information on the electric and magnetic contrasts near subwavelength dielectric particles. For example, for the electric contrast, we can write

$$
\Xi_{e, s}=-\frac{2 \alpha_{\|}}{\left(Z_{0}-R\right)^{3}}\left\{1+o\left(\left|Z_{0}-R\right|^{6}\right)\right\}
$$

and

$$
\Xi_{e, p}=\frac{2 \alpha_{\perp}\left(n^{2} \sin ^{2} \theta_{0}+1\right)}{\left(n^{2} \sin ^{2} \theta_{0}-1\right)\left(Z_{0}-R\right)^{3}}\left\{1+o\left(\left|Z_{0}-R\right|^{6}\right)\right\}
$$

The main physical behaviors predicted by these relations as well as the two ones for the magnetic part are summarized in tables I and II.

Before discussing further the physical meaning of these tables, let us note that the trends gathered in tables I and II, on the basis of the elementary model developped in this section, are in agreement with the outputs of various the Maxwell equations solvers designed to cope with nanoscopic parameters ${ }^{31,35}$. Specifically, the technique of perturbation in reciprocal space $^{69}$, based on the Rayleigh approximation, leads to the same conclusions.

The validity of the above theoretical analysis is supported by the successfull explanation of the contrasts observed by passive probe devices. As detailed in the next section, when dealing with subwavelength sized localized objects, the contrasts predicted by the simple dipolar model are found in excellent agreement with available PSTM experimental data on relevant dielectric objects ${ }^{70}$.

We close this section by noticing that, in the $p$ polarized mode and close to subwavelength size dielectric objects, equation(31) predicts strongly contrasted dark magnetic field intensity 
patterns which dramatically depends on the incident illumination angle $\theta_{0}$ (see table II). Although the complete experimental investigation of this peculiar effect has not been yet performed, this trends seems to be in qualitative agreement with recent local measurements of the optical magnetic field using Au coated tips ${ }^{71}$.

\section{PASSIVE PROBE DEVICES: DIRECT COMPARISON OF THEORETICAL AND EXPERIMENTAL IMAGES}

The point of view developped in section II states that images recorded by passive probe devices should be interpreted as the result of the scattering of quasi-two-dimensional surface waves by the various defects (asperities, kink, etc...) lying on the sample surface. The surface waves generated by total internal reflection are viewed as quasi-two-dimensional because they decay exponentially in the direction normal to the sample surface. The surface defects scatter these waves parallel to the surface and induce some loss due to diffraction which couples light to the external medium. Passive probe near-field optical microscopes approach a local probe very close to the sample surface so that they detect mostly the field distribution of the quasi-two-dimensional scattered surface wave rather than the diffracted components which propagate away from the sample surface.

\section{A. Dielectric samples}

Using passive probe devices, the observation of dielectric structures deposited on dielectric substrates allows to check experimentally the validity of the analysis of section IV C. Such experimental test was performed recently ${ }^{70}$ using a sample displaying sub-wavelength features. The patterns consist of several glass pads lying on a glass surface (figure 4) obtained by standard electron beam lithography and reactive ion etching techniques. In the sample of figure 4 (a), six pads had initially been placed at the corners of an hexagon around a central pad. Unfortunately, the lower left pad was later lost during the ultrasonic cleaning process. Also, a large aggregate (approximately $400 \mathrm{~nm} \times 300 \mathrm{~nm}$ ) is found in the lower 
right part of the scanned area. Nevertheless, this defect is useful to support the explanation of the optical effect to be discussed below.

Figures 5 and 6 show constant intensity PSTM images recorded with sharpened bare (uncoated) optical fibers for both incident polarizations $s$ et $p$. The angle of incidence of 60 degrees and the incident wavelength $\lambda=633 \mathrm{~nm}$ set the effective wavelength $\lambda_{\|}$of the surface wave close to $500 \mathrm{~nm}$.

Figure 5 displays the image recorded close to the sample of figure 4 (a). In both polarizations, the field detected above the pads appears bright but the multiple scattering of the incident surface wave downgrades the image-object relation . This multiple scattering is not only due to the pads themselves but also to other structures or defects located at larger distances outside of the scan area of the image. For surface structures of the order of $\lambda_{\|} / 2$ (mesoscopic regime), it thus turns out that the multiple scattering of the quasi-twodimensional waves builds the dominant feature of the image recorded by a passive probe device. This means that the image provides informations about the spatial distribution of the optical field rather than a direct identification of the shapes of the underlying objects. Such observation is thus in agreement with the point (a) of section II E.

Let us now consider the sample of figure 4 (b) where the pads size is of the order of $\lambda_{\|} / 5$. In this nanoscopic regime, recent theoretical works ${ }^{72}$ demonstrated that reducing the defects and their relative spacing to nanoscopic sizes leads to the extinction of the interferences due to the multiple scattering of the surface waves and a concurrent increasing importance of the light confinement effects summarized in tables I and II. When the fabricated nanoscopic structures were illuminated under TM polarization, they showed a positive (bright) contrast in the near-field image (Fig. 6 (a)). But they appear with a negative (dark) contrast if the incident field is TE polarized (Fig. 6 (b)). Observing this inversion of contrast is associated with the nanoscopic size of the pads. Indeed, the larger defect in the lower right part of the scanned area (see figure 4 (b) reference) is recognized by a positive contrast in both polarizations. If we now check against the dependence of the contrast on the incident polarization in tables I and II, we deduce that the sharpened bare (uncoated) optical fibers 
detect signals of electric nature. On the basis of this hint about the nature of the signal, it is now possible to answer to the question (a) of section II E by computing exactly the nearfield spatial distribution of $|\mathbf{E}(\mathbf{r}, \omega)|^{2}$, in the absence of the tip, in order to see if it matches the experimental images of figure 6. Taking into account the experimental illumination conditions, the computations presented in figure 7 have solved exactly the discretized form of equation (5) where the experimental topography of figure 4 (b) defines the input values of $\chi\left(\mathbf{r}^{\prime}, \omega\right)$. The excellent agreement between the experimental results and the theoretical distribution of $|\mathbf{E}(\mathbf{r}, \omega)|^{2}$, as computed in the absence of the tip, supports a positive answer to the question (a) of section IIE. Let us notice that the translation of the features of the computed image relatively to the ones of the experimental image is explained by the fact that the AFM topographical data used as inputs of the computations are not centered at the same location of the sample surface as the PSTM data: indeed, after substituting the AFM tip by the sharpened optical fiber, it is not possible to scan exactly the same area.

We close this section by mentionning that signals of magnetic nature have been discovered when using Au coated fiber tips in a passive probe device ${ }^{71}$. Works in progress confirm that this kind of tips may indeed lead to the detection of the distribution of the magnetic field associated to the optical wave.

\section{B. Resonant metallic structures}

Still using passive probe device, we now turn to the observation of metallic nanostructures sustaining plasmon resonances ${ }^{73}$. The resonant feature of these nanostructures is associated with an optical response which does not meet the criterion of equation (25). The theoretical analysis of section IV C does not apply anymore so that we will compare the experimental results directly with the numerical solution of equation (5).

Also resulting of a microfabrication process, Au particles are deposited on ITO glass substrate. Their geometry was checked by scanning electron microscopy and AFM measurements. The particles shape was found to be akin of a half oblate spheroid (cut plane on 
the substrate). Their typical sizes were a $100 \times 100 \mathrm{~nm}^{2}$ section and a $40 \mathrm{~nm}$ height. A microspectrometer located in the far-field measured the single particles' plasmon peak at the wavelength of $640 \mathrm{~nm}$.

The plasmon resonance of the Au particles is associated to a large lateral variation of the intensity in the near-field zone so that the image acquisition can not be performed efficiently at constant intensity. The tip is thus scanned at a constant height above the sample surface while monitoring the light intensity level. Successive images are recorded while bringing the tip closer to the sample surface by $5 \mathrm{~nm}$ steps. The series of images terminates when the tip touches a particle. One retains the last complete image which was recorded before the tip crash.

In the computation which does not include any tip, the $p$ polarized incoming field $\mathbf{E}_{0}$ $(\lambda=633 \mathrm{~nm})$ was adjusted to fit the experimental conditions: total internal reflection with an angle of incidence equal to 55 degrees and projection of the incident wavevector onto the surface substrate pointing mostly towards the $y$ direction with a slight tilt to the upper left corner of the images. To solve the discretized form of equation (5), the discretization cells of a single $\mathrm{Au}$ particle were chosen to be $10 \times 10 \times 10 \mathrm{~nm}^{3}$ cubes. On the basis of the results found for dielectric samples, the use of sharpened bare (uncoated) optical fiber tips leads to compare the experimental image with the theoretical distribution of $|\mathbf{E}(\mathbf{r}, \omega)|^{2}$.

Figure 8 compares a theoretical computation with an experimental PSTM image recorded above an isolated Au particle. Fig. 8 (b) maps the distribution of the normalized intensity of the electric field associated to the optical wave $\left(|\mathbf{E}(\mathbf{r}, \omega)|^{2} /\left|\mathbf{E}_{0}(\mathbf{r}, \omega)\right|^{2}\right)$ at the constant height $z=140 \mathrm{~nm}$ above the glass substrate. This constant height does not correspond to the experimental tip to sample distance which was determined to be less than $45 \mathrm{~nm}$ since the experimental image displayed in Fig. 8 was the last one recorded before the tip touched the particle due to approaching the tip $5 \mathrm{~nm}$ closer to the sample. The height $z=140 \mathrm{~nm}$ was found to provide the best agreement between the computed image and the experimental one after examining systematically the computed distribution patterns in planes parallel to the substrate surface at heights ranging between 0 and $200 \mathrm{~nm}$. Since, in this height range, 
the distribution patterns are very similar from one plane to another, the best agreement is defined as the pattern exhibiting a contrast between the lowest and the highest intensity which is closest to the experimental image. We justify this procedure by the fact that the tip is not included in the calculation. Since the tip integrates the optical field over a certain volume, the heigth $z=140 \mathrm{~nm}$ should be interpreted as a rough modeling of the averaging process occuring inside the tip.

The agreement between the patterns of the calculated and the experimental images is excellent. Since the simulation has not included the tip, the experimental image exhibits a broader and less contrasted pattern. The simulation recovers successfully the interference between the incident surface optical wave and the wave scattered by the Au particle. The computation fixes the exact position of the particle to be between the two central bright spots. The near-field optical signature of a single Au resonant nanoparticle, as detected by a passive probe device, is thus different of the signature of the dielectric structures with similar geometrical features which was discussed in the preceding section. Moreover, this signature turn out to be much larger than the size of the underlying resonant Au particle. Once again, all these features support the point of view that active probe device provide image of the spatial distribution of $|\mathbf{E}(\mathbf{r}, \omega)|^{2}$.

\section{Observation of localized plasmons coupling}

To give a definitive positive answer to the question (a) of section (II-E) one can use an arrangement of $\mathrm{Au}$ particles, identical to the ones described in the preceding section, in order to build a different distribution $|\mathbf{E}(\mathbf{r}, \omega)|^{2}$ to be detected by the near-field microscope. This can be achieved by aligning 10000 of such particles in a row with a spacing of 100 nm. In this configuration, the localized plasmons of each individual particle may couple to each other and set a hybrid plasmon mode. Using the same illumination and detection conditions as in the preceding section, the observation of this phenomenon is reported in figure 9 (a). The main feature of this coupling is the transverse squeezing of the optical field 
distribution which contrasts with the broad spots found above a single particle (see figure 8). Indeed, the tip has integrated the detection of the optical field over its own volume at least. Consequently, the field distribution in the absence of the tip is probably narrower. The model calculation (figure 9 (b)) confirms that the spots are narrower than in the case of the single $\mathrm{Au}$ particle (figure 8). However, the calculated squeezing is not so narrow as in the experiment since the modeling involved only 30 particles instead of the 10000 present in the experiment. The squeezing probably increases as the chain length grows.

\section{ACTIVE PROBE DEVICES:}

\section{THEORETICAL ASSESSMENT OF THE RELEVANCE OF THE LDOS}

A rapid overview of the near-field optics literature brings easily to the fore that the ideal active probe device is considered to be a point-like source of light. Various empirical procedures intend to produce tips which are as close as possible to this ideal emitting feature. A fluorescing structure (made of one or several molecules) attached at the apex of a tip

provides a good approximation to such ideal point source ${ }^{74}$. Therefore, we will tackle the theoretical assessment of the relevance of the electromagnetic LDOS to interpret the images delivered by active probe devices using the model of a fluorescent structure as local emitter of light. This choice presents not only the advantage to weaken the need for a discussion about the most appropriate tip shape and size which enter the calculation, but is also of actual experimental interest: indeed, six years ago, the detection of local signals emitted by individual molecules was reported by Betzig and Chichester ${ }^{75}$. In particular, these authors demonstrated how a fluorescing individual molecule was able to map the electric field intensity generated at the apex of a metal coated tip. Since these pionneering work, an increasing interest has been devoted to single molecular detection technique ${ }^{76}$. 


\section{A. Molecules in confined geometry}

In the presence of molecules, the optical electric field calculation turns out to be a difficult task, and different approximations may be applied ${ }^{77-82}$. Several microscopic systems (adsorbates) in interaction with a mesoscopic surrounding (like the tip-surface junction) build a low symmetry system and, consequently, makes the application of any method based on standard boundary condition particularly heavy. For our purpose, we will show that the basic theory developped in section III provides an appropriate framework to discuss some theoretical aspects of the emission of light by a fluorescing molecule placed in a complex surrounding. Starting from a standard light-matter coupling Hamiltonian, we derive a selfconsistent equation for the optical field lying around molecular systems. At the fluorescence wavelength, we will establish the direct relation existing between the fluorescence lifetime change and the electromagnetic local density of state associated with the sample.

\section{B. Quantum derivation of the self-consistent field}

Let us consider a single molecule trapped inside a confined geometry (cf. figure 10). For example, it may be the junction formed by the tip of a scanning near field optical microscope (SNOM) facing the surface of a sample. When this system is perturbed by an external optical field $\mathbf{E}_{0}(\mathbf{r}, \omega)$ coming from an external laser source, the field $\mathbf{E}(\mathbf{r}, \omega)$ in the gap may be derived from the formalism described in section III. We temporarily switch from the $\omega$-space to the time dependent representation and note that the field $\mathbf{E}$ is an observable physical quantity since it has already been averaged on the quantum states of the whole primary system (dielectric surrounding).

The interaction Hamiltonian coupling between the primary system and the molecule reads

$$
H(t)=-\left(\mathbf{E}\left(\mathbf{r}_{m}, t\right)+\mathcal{E}\left(\mathbf{r}_{m}, t\right)\right) \cdot \mu(t),
$$

where $\mu(t)$ and $\mathcal{E}\left(\mathbf{r}_{m}, t\right)$ are, respectively, the polarization operator of the molecule and the 
electric field operator associated with the three-dimensional dielectric surrounding. The vector $\mathbf{r}_{m}$ labels the position of the molecule. These operators are written here in the interaction representation

$$
\mathcal{E}\left(\mathbf{r}_{m}, t\right)=\exp \left[i \hbar^{-1} H_{0} t\right] \mathcal{E}\left(\mathbf{r}_{m}\right) \exp \left[-i \hbar^{-1} H_{0} t\right]
$$

and

$$
\mu(t)=\exp \left[i \hbar^{-1} H_{0} t\right] \mu \exp \left[-i \hbar^{-1} H_{0} t\right]
$$

where $H_{0}$ represents the Hamiltonian for the tree-dimensional dielectric surrounding. As in all standard linear response calculations, we consider that the perturbation is limited to one that vanishes sufficiently as $t \rightarrow-\infty$. To ensure the adiabatic turning on of the perturbation we replace $H(t)$ by $H(t) \exp ^{\eta t}$ where $\eta$ is greater than zero, but vanishingly small. Note that in the present description, the eigenstates associated with the field operator $\left.\mathcal{E}\left(\mathbf{r}_{m}, t\right)\right)$ are the eigenstates of the dielectric surrounding.

The simultaneous time evolution of both field states and molecular states can be derived by well-established techniques based on time-dependent perturbations theories. In the interaction representation, the wave function $\mid \psi(t)>$ that describes the evolution of the whole system is given by

$$
\left|\psi(t)>=\exp \left[i \hbar^{-1} H_{0} t\right]\right| \phi(t)>
$$

where $\mid \phi(t)>$ labels the wave function of the entire system written in the Schrödinger representation. A first order expansion of $\mid \psi(t)>$ leads to the well-known result

$$
|\psi(t)>=| \phi_{0}(t)>+\frac{1}{i \hbar} \int_{-\infty}^{t} H\left(t^{\prime}\right) \mid \phi_{0}\left(t^{\prime}\right)>d t^{\prime}
$$

\section{Quantum Average of the Optical Field}

At this stage, since one neglects all chemical interactions between the molecule and its surrounding, one can assume that there is no significant modification of the whole wave 
function $\mid \psi>$ of the system due to the short range interaction (of chemical origin for example) between the molecule and the tree-dimensional dielectric surrounding. In this situation, it is worthwhile to apply the time dependent Hartree approximation in which one assumes that each part of the system moves under the combined effect of the external force and the average displacement of the other system. A straightforward application of the perturbation theory shows that the linear response of the field $\mathcal{E}\left(\mathbf{r}_{m}, t\right)$ at the location of the molecule is given by

$$
\begin{array}{r}
\mathbf{E}_{m}\left(\mathbf{r}_{m}, t\right)=<\mathbf{E}\left(\mathbf{r}_{m}, t\right)+\mathcal{E}\left(\mathbf{r}_{m}, t\right)>=\mathbf{E}\left(\mathbf{r}_{m}, t\right) \\
+\int_{-\infty}^{t} \mathbf{S}\left(\mathbf{r}_{m}, \mathbf{r}_{m}, t-t^{\prime}\right) \cdot<\mu\left(t^{\prime}\right)>d t^{\prime}
\end{array}
$$

In this time-dependent equation, the dyadic $\mathbf{S}\left(\mathbf{r}_{m}, \mathbf{r}_{m}, t-t^{\prime}\right)$ represents merely the timedependent field-susceptibility associated with the SNOM junction (the $\omega$-space representation of $\mathbf{S}$ appears in equation 11). From relations (34) to (39), it is clear that $\mathbf{S}$ is formed by a succession of quadratic products of the different matrix elements of the operator $\mathcal{E}\left(\mathbf{r}_{m}\right)$

$$
\begin{aligned}
& \mathbf{S}\left(\mathbf{r}, \mathbf{r}^{\prime}, t-t^{\prime}\right)= \\
& \frac{i}{\hbar} \sum_{m, n} \rho_{m, m}\left\{\mathcal{E}(\mathbf{r})_{m n} \mathcal{E}\left(\mathbf{r}^{\prime}\right)_{n m} e^{i / \hbar\left(E_{n}-E_{m}\right)\left(t-t^{\prime}\right)}\right. \\
&\left.-\mathcal{E}\left(\mathbf{r}^{\prime}\right)_{m n} \mathcal{E}(\mathbf{r})_{n m} e^{-i / \hbar\left(E_{n}-E_{m}\right)\left(t-t^{\prime}\right)}\right\}
\end{aligned}
$$

where $E_{m}$ and $E_{n}$ are energies of the unperturbed quantum states of the surrounding and $\rho_{m, m}$ a diagonal element of the density matrix. In equation (39), the quantum average $<\mu(t)>$ can be expressed by a second linear equation

$$
<\mu(t)>=\int_{-\infty}^{t} \alpha_{m}\left(t-t^{\prime}\right) \cdot \mathbf{E}_{m}\left(\mathbf{r}_{m}, t^{\prime}\right) d t^{\prime}
$$

where $\alpha_{m}\left(t-t^{\prime}\right)$ is the time-dependent molecular polarizability

$$
\begin{array}{r}
\alpha_{m}\left(t-t^{\prime}\right)=\frac{i}{\hbar} \sum_{r, s} \rho_{r, r}\left\{\mu_{r s} \mu_{s r} e^{i / \hbar\left(E_{r}-E_{s}\right)\left(t-t^{\prime}\right)}\right. \\
\left.-\mu_{r s} \mu_{s r} e^{-i / \hbar\left(E_{r}-E_{s}\right)\left(t-t^{\prime}\right)}\right\} .
\end{array}
$$


In the above equation, $E_{r}$ and $E_{s}$ are energies of the unperturbed quantum states of the molecule. From these relations one may write the time-dependent self-consistent equation governing both the magnitude and the polarization of the optical molecular field

$$
\begin{gathered}
\mathbf{E}_{m}\left(\mathbf{r}_{m}, t\right)=\mathbf{E}\left(\mathbf{r}_{m}, t\right)+\int_{-\infty}^{t} d t^{\prime} \int_{-\infty}^{t^{\prime}} d t^{\prime \prime} \\
\mathbf{S}\left(\mathbf{r}_{m}, \mathbf{r}_{m}, t-t^{\prime}\right) \cdot \alpha_{m}\left(t^{\prime}-t^{\prime \prime}\right) \cdot \mathbf{E}_{m}\left(\mathbf{r}_{m}, t^{\prime \prime}\right) .
\end{gathered}
$$

Solving this implicit integral equation is possible in the $\omega$-space

$$
\begin{aligned}
\mathbf{E}_{m}\left(\mathbf{r}_{m}, \omega\right) & =\mathbf{E}\left(\mathbf{r}_{m}, \omega\right) \\
& +\mathbf{S}\left(\mathbf{r}_{m}, \mathbf{r}_{m}, \omega\right) \cdot \alpha_{m}(\omega) \cdot \mathbf{E}_{m}\left(\mathbf{r}_{m}, \omega\right)
\end{aligned}
$$

with

$$
\alpha_{m i, j}(\omega)=\frac{1}{\hbar} \sum_{r, s} \rho_{r r}\left\{\frac{\mu_{r s}^{i} \mu_{s r}^{j}}{\omega-\omega_{s r}-i \Gamma_{s r}}-\frac{\mu_{r s}^{j} \mu_{s r}^{i}}{\omega+\omega_{s r}-i \Gamma_{s r}}\right\}
$$

In this relation, we have introduced the dampings $\Gamma_{s r}$ by applying the prescription of Ward ${ }^{83}$. When one considers just a single molecule, equation (44) is very easy to solve. We obtain then relations similar to those already exposed in section IV. The main difference lies into the choice of the propagator $\mathbf{S}$ which, in the present case must be associated with the complete SNOM junction

$$
\mathbf{E}_{m}\left(\mathbf{r}_{m}, \omega\right)=\mathbf{M}\left(\mathbf{r}_{m}, \omega\right) \cdot \mathbf{E}\left(\mathbf{r}_{m}, \omega\right)
$$

where $\mathbf{M}\left(\mathbf{r}_{m}, \omega\right)$ is a $(3 \times 3)$ matrix defined by

$$
\mathbf{M}\left(\mathbf{r}_{m}, \omega\right)=\left[\mathbf{I}-\mathbf{S}\left(\mathbf{r}_{m}, \mathbf{r}_{m}, \omega\right) \cdot \alpha_{m}(\omega)\right]^{-1}
$$

Note that, from this equation one obtains in a first stage, the molecular effective field $\mathbf{E}_{m}\left(\mathbf{r}_{m}, \omega\right)$. The field $\mathbf{E}_{m}(\mathbf{r}, \omega)$ generated by the molecule far away from the emitting zone can be described by applying once again the Lippmann-Schwinger equation with a single scattering center (the molecule) 


$$
\begin{aligned}
& \quad \mathbf{E}_{m}(\mathbf{r}, \omega)=\mathbf{E}(\mathbf{r}, \omega) \\
& +\mathbf{S}\left(\mathbf{r}, \mathbf{r}_{m}, \omega\right) \cdot \alpha_{m}(\omega) \cdot \mathbf{M}\left(\mathbf{r}_{m}, \omega\right) \cdot \mathbf{E}_{0}\left(\mathbf{r}_{m}, \omega\right)
\end{aligned}
$$

that can be rewritten as

$$
\begin{aligned}
\mathbf{E}_{m}(\mathbf{r}, \omega) & =\mathbf{E}(\mathbf{r}, \omega) \\
& +\mathbf{S}\left(\mathbf{r}, \mathbf{r}_{m}, \omega\right) \cdot \alpha\left(\mathbf{r}_{m}, \omega\right) \cdot \mathbf{E}\left(\mathbf{r}_{m}, \omega\right)
\end{aligned}
$$

where, in complete analogy with the point-like surface defect treated in section IV (equation 20), $\alpha\left(\mathbf{r}_{m}, \omega\right)$ defines the effective polarizability of the molecule interacting with its dielectric environment.

$$
\alpha\left(\mathbf{r}_{m}, \omega\right)=\alpha_{m}(\omega) \cdot \mathbf{M}\left(\mathbf{r}_{m}, \omega\right)
$$

\section{Lifetime change and electromagnetic LDOS}

The molecule radiates optical energy with a polarizability $\alpha\left(\mathbf{r}_{m}, \omega\right)$ "dressed" by the dielectric surrounding. The symmetry of the tensor $\alpha\left(\mathbf{r}_{m}, \omega\right)$ is governed mainly by the symmetry of the molecule-surrounding super system even if the molecular polarizability $\alpha_{\mathbf{0}}(\omega)$ is initially isotropic. For example, in the particular case of a single molecule interacting with a perfectly planar surface, the dyadic tensor $\alpha\left(\mathbf{r}_{m}, \omega\right)$ belongs to the $C_{\infty v}$ symmetry group so that it may also be described with only two independent components $\alpha_{\|}$and $\alpha_{\perp}$. In the case of a SNOM surrounding (corrugated surface + pointed detector), the effective polarizability tensor may be more complex. The new molecular lifetime $\Gamma^{-1}\left(\mathbf{r}_{m}\right)$ is contained implicitly in this tensor.

Two important electrodynamical effects appear in the effective polarizability. The first is a small shift of the excited state: the fluorescence frequency $\omega_{0}$ is shifted towards a lower frequency $\omega_{\text {eff }}$. This effect can be characterized by the ratio

$$
\Omega\left(\mathbf{r}_{m}\right)=\omega_{e f f} / \omega_{0}
$$


Quite often, this coefficient is close to unity. For our purpose, we may safely neglect its eventual variation. The second effect, much more sensitive to the location of the molecule in the junction, is the well-known lifetime change defined by the ratio

$$
\eta\left(\mathbf{r}_{m}\right)=\Gamma^{-1}\left(\mathbf{r}_{m}\right) / \Gamma_{0}^{-1}
$$

where $\Gamma_{0}$ represents the molecular linewidth of the isolated molecule. This coefficient also depends on the effective polarizability component. To proceed further analytically, we assume, with a good approximation, that the interacting molecule embedded in the SNOM surroundings still displays an effective polarizability belonging to the $C_{\infty v}$ group with its main axis along the $z$ direction. The two independent components $\alpha_{\perp}\left(\mathbf{r}_{m}, \omega\right)$ and $\alpha_{\|}\left(\mathbf{r}_{m}, \omega\right)$ may then be identified to a two-levels polarizability expression similar to the one of an isolated molecule

$$
\alpha_{\perp}\left(\mathbf{r}_{m}, \omega\right)=\left\{\frac{2 \omega_{\perp} A_{\perp}}{\omega_{\perp}^{2}-\omega^{2}-i \omega \Gamma_{\perp}}\right\}=\alpha_{m}(\omega) \mathbf{M}_{z z}\left(\mathbf{r}_{m}, \omega\right)
$$

and

$$
\alpha_{\|}\left(\mathbf{r}_{m}, \omega\right)=\left\{\frac{2 \omega_{\|} A_{\|}}{\omega_{\|}^{2}-\omega^{2}-i \omega \Gamma_{\|}}\right\}=\alpha_{m}(\omega) \mathbf{M}_{x x}\left(\mathbf{r}_{m}, \omega\right)
$$

For each equation, the three parameters $\omega_{\perp}, A_{\perp}$ and $\Gamma_{\perp}$ (respectively $\omega_{\|}, A_{\|}$and $\left.\Gamma_{\|}\right)$can be identified by introducing the two-level representation of $\alpha_{m}(\omega)$

$$
\alpha_{m}(\omega)=\frac{A_{0} \omega_{0}}{\omega_{0}^{2}-\omega^{2}-i \omega \Gamma_{0}}
$$

where $A_{0}=\frac{2 \mu^{01} \mu^{10}}{\hbar}$.

One then finds ( $\Re$ denotes the real part):

$$
\Gamma_{\perp / \|}=\Re \frac{\Gamma_{0} \mathbf{M}_{x x / z z}\left(\mathbf{r}_{m}, 0\right)}{\mathbf{M}_{x x / z z}\left(\mathbf{r}_{m}, \omega_{0}\right)}
$$

or, similarly:

$$
\eta\left(\mathbf{r}_{m}\right)=\Re \frac{\mathbf{M}_{x x / z z}\left(\mathbf{r}_{m}, 0\right)}{\mathbf{M}_{x x / z z}\left(\mathbf{r}_{m}, \omega_{0}\right)}
$$


where the notation $x x / z z$ means that the element $x x$ of $\mathbf{M}$ is required to compute $\Gamma_{\perp}$ while the element $z z$ is used to compute $\Gamma_{\|}$.

In conclusion, one obtains a compact result, depending only on the real part of the dynamical matrix $\mathbf{M}\left(\mathbf{r}_{\mathbf{m}}, \omega_{0}\right)$ at the resonance frequency of the molecule. Note that the spatial variation of $\eta\left(\mathbf{r}_{m}\right)$ with respect to the dielectric surroundings is contained in the field-susceptibility $\mathbf{S}\left(\mathbf{r}_{m}, \mathbf{r}_{m}, \omega\right)$. In the vicinity of a highly complex system, this dyadic may be obtained by solving the Dyson equation (11).

A link with the electromagnetic LDOS arises explicitly when the matrix $\mathbf{M}$ is expanded to the first Born approximation:

$$
\mathbf{M}\left(\mathbf{r}_{m}, \omega\right)=\mathbf{I}+\alpha_{0}(\omega) \cdot \mathbf{S}\left(\mathbf{r}_{m}, \mathbf{r}_{m}, \omega\right)
$$

Using the two-level representation of $\alpha_{m}$ allows to write

$$
\mathbf{M}_{x x / z z}\left(\mathbf{r}_{m}, \omega_{0}\right)=1+i \frac{A_{0}}{\Gamma_{0}} \mathbf{S}_{x x / z z}\left(\mathbf{r}_{m}, \mathbf{r}_{m}, \omega_{0}\right)
$$

whose the real part is given by

$$
\Re\left[\mathbf{M}_{x x / z z}\left(\mathbf{r}_{m}, \omega\right)\right]=1-\frac{A_{0}}{\Gamma_{0}} \Im\left[\mathbf{S}_{x x / z z}\left(\mathbf{r}_{m}, \mathbf{r}_{m}, \omega_{0}\right)\right]
$$

For the effective width of the excited molecular level, this approximation leads to

$$
\Gamma_{\| / \perp}=\Gamma_{0}+A_{0} \Im\left[\mathbf{S}_{x x / z z}\left(\mathbf{r}_{m}, \mathbf{r}_{m}, \omega_{0}\right)\right]
$$

This result was also derived by Metiu within a classical approach ${ }^{17}$. Several remarks may be done about these relations. First, the propagator $\mathbf{S}$ of the environment controls the molecular lifetime change. From a practical point of a view, it may be accurately modeled for any kind of surface profiles and materials by solving the Dyson equation (11). For realistic configurations this purpose needs a numerical procedure based, for example, on the recursive algorithm described in references ${ }^{84,85,77}$.

Second, the propagator $\mathbf{S}\left(\mathbf{r}_{m}, \mathbf{r}_{m}, \omega_{0}\right)$ is very sensitive to the molecule-sample distance variation. Consequently the molecular lifetime change constitutes a highly sensitive nanoprobe to the external environment. In particular, working in the near-field zone just 
before the quenching effect occurs, should make possible to increase the lateral SNOM resolution ${ }^{77,78,81}$.

Third, equation (61) indicates that the spontaneous molecular decay rate is modified by any changes in the electromagnetic local density of states with respect to LDOS of vacuum (implicitly contained in the natural linewidth $\Gamma_{0}$ ). Indeed, if we introduce the average effective line width

$$
\Gamma\left(\mathbf{r}_{m}\right)=\frac{1}{3}\left(2 \Gamma_{\|}+\Gamma_{\perp}\right)
$$

we can write

$$
\Gamma\left(\mathbf{r}_{m}\right)=\Gamma_{0}+\frac{\pi \omega_{0}^{2} A_{0}}{c^{2}} \rho\left(\mathbf{r}_{m}, \omega_{0}\right)
$$

where $\rho\left(\mathbf{r}_{m}, \omega_{0}\right)$ has been defined in equation (8).

At a given position, a larger LDOS, which means that a growing number of electromagnetic states are available, is responsible for shorter lifetime of the molecule. Let us now imagine that the fluorescent structure scans a sample surface. Referring to the classification of active probe devices introduced in section II D, such structure may be viewed as an ideal point source. According to equation (63), scanning a fluorescent structure should image the electromagnetic LDOS. To illustrate what kind of information can then be extracted from $\Gamma\left(\mathbf{r}_{m}\right)$, figure 11 presents LDOS maps close to a system of nanoscopic glass pads deposited on a flat glass surface. The symmetry of the pads arrangement builds a specific LDOS pattern where maxima may occur at other locations than right above the pads (figure 11 (B)). Similarly to the case of passive probe devices, one can then expect that an active probe device provides an image which does not allow a direct recognition of the shape of the underlying objects. Indeed, as in the exemple of figure 11, the LDOS may display significant discrepancies relatively to the shapes of the underlying objects. 


\section{CONCLUSION}

On the basis of a simple classification of near-field optical microscope into passive and active probe devices, this review focuses on the crucial question of the interpretation of the images recorded by these microscopes. The paper further proposes a practical point of view which, according to the passive or active character of the probe tip, leads to formulate different hypothesis about the nature of the information contained in near-field optical images. The practical point of view suggests that the distribution of the electromagnetic near-field scattered by the sample surface is probed by passive probe device while the electromagnetic local density of states might be the key to understand the images recorded by active probe devices. For both kinds of devices, this means that the resulting contrasts do not necessarily help to recognize directly the shape of the underlying objects.

In the case of passive probe devices, the direct comparison of experimental and theoretical results supports the proposed point of view. It also brings to the fore that a combined use of several techniques as electron-beam lithography, numerical simulations and direct observation of near-field phenomena is an effective procedure to improve the understanding of near-field optical effects. We suggest that the same kind of procedure should be applied to establish whether images recorded with active probe devices should be interpreted on the basis of the electromagnetic LDOS or not.

An important perspective, issued from the interpretation scheme proposed in this paper, is the development of a new class of near-field optics experiments which intend to use the optical tunnel effect in order to control, on a submicrometer scale, the optical energy transfer between several delocalized detection or injection centers ${ }^{86^{-88,73,89}}$.

\section{ACKNOWLEDGMENTS}

Stimulating discussions and/or collaborations with F. R. Aussenegg, J. R. Krenn, A. Leitner, G. Schider, U. Ch. Fischer, E. Bourillot, T. David, C. Chicanne, E. Devaux, J. P. 
Goudonnet and Y. Lacroute are gratefully acknowledged. One of us (A. D.) acknowledges the financial support of the Regional Council of Burgundy. 


\section{REFERENCES}

${ }^{1}$ G. S. Agarwal, Phys. Rev. A 11, 230 (1975).

${ }^{2}$ E. A. Ash and G. Nicholls, Nature 237, 510 (1972).

${ }^{3}$ P. R. Antoniewicz, Phys. Rev. Let. 32, 1424 (1974).

${ }^{4}$ H. A. Bethe, Phys. Rev. 66, 163 (1944).

${ }^{5}$ C. K. Carniglia, L. Mandel, and K. H. Drexhage, J. Opt. Soc. Am. 62, 479 (1972).

${ }^{6}$ V. Celli, A. Marvin, and F. Toigo, Phys. Rev. B 11, 1779 (1975).

${ }^{7}$ K. L. Kliewer and R. Fuchs, Advances in Chemical Physics 27, 355 (1974).

${ }^{8}$ W. Lukosz and R. E. Kunz, J. Opt. Soc. Am. 67, 1607 (1977).

${ }^{9}$ C. Mavroyannis, Mol. Phys. 6, 593 (1963).

${ }^{10}$ A. D. Maclachlan, R. D. Gregory, and M. A. Ball, Mol. Phys. 7, 119 (1963).

${ }^{11}$ J. Mahanty and B. W. Ninham, J. Chem. Phys. 59, 6157 (1973).

12 A. A. Maradudin and W. Zierau, Phys. Rev. B 14, 484 (1976).

${ }^{13}$ A. Otto, Zeitschrift für Physik 216, 398 (1968).

${ }^{14}$ R. Ruppin, Surf. Sci. 34, 20 (1973).

${ }^{15}$ S. Efrima and H. Metiu, J. Chem. Phys. 70, 1939 (1979).

${ }^{16}$ T. Maniv and H. Metiu, J. Chem. Phys. 76, 696 (1982).

${ }^{17}$ H. Metiu, Progress in Surface Science 17, 153 (1984).

${ }^{18}$ D. W. Pohl, W. Denk, and M. Lanz, Appl. Phys. Lett. 44, 651 (1984).

${ }^{19}$ E. Betzig, A. Harootunian, A. Lewis, and M. Isaacson, Appl. Opt. 25, 1890 (1986).

${ }^{20}$ E. Betzig, M. Isaacson, and A. Lewis, Appl. Phys. Lett. 51, 2088 (1987). 
${ }^{21}$ E. Betzig et al., Science 251, 1468 (1991).

${ }^{22}$ R. C. Reddick, R. J. Warmack, and T. L. Ferrell, Phys. Rev. B 39, 767 (1989).

${ }^{23}$ D. Courjon, K. Sarayedine, and M. Spajer, Opt. Comm. 71, 23 (1989).

${ }^{24}$ N. F. van Hulst, F. B. Segerink, F. Achten, and B. Bolger, Ultramicroscopy 42, 416 (1992).

${ }^{25}$ N. F. van Hulst, M. H. Moers, and B. Bölger, J. of Microscopy 171, 95 (1993).

${ }^{26}$ in Near-field optics, Vol. E 242 of NATO ASI, NATO, edited by D. Pohl and D. Courjon (Kluwer, Dordrecht, 1993).

${ }^{27}$ A. G. Choo et al., Appl. Phys. Let. 65, 947 (1994).

${ }^{28}$ P. Dawson, F. de Fornel, and J. P. Goudonnet, Phys. Rev. Lett. 72, 2927 (1994).

${ }^{29}$ A. Gustafsson, M. E. Pistol, L. Montelius, and L. Samuelson, J. Appl. Phys. 84, 1715 (1998).

${ }^{30}$ S. I. Bozhevolnyi and B. Vohnen, Phys. Rev. Let. 77, 3351 (1996).

${ }^{31}$ C. Girard and A. Dereux, Report on Progress in Physics 59, 657 (1996).

${ }^{32}$ B. Hecht et al., J. Appl. Phys. 81, 2492 (1997).

${ }^{33}$ N. Garcia and M. Nieto-Vesperinas, Opt. Lett. 18, 2090 (1993).

${ }^{34}$ R. Carminati and J. J. Greffet, Opt. Comm. 116, 316 (1995).

${ }^{35}$ J.-J. Greffet and R. Carminati, Progress in Surface Science 56, 133 (1997).

${ }^{36}$ A. Zangwill, Physics at surfaces (Cambridge University Press, New York, 1988).

${ }^{37}$ D. Courjon and C. Bainier, Rep. Prog. Phys. 57, 989 (1994).

${ }^{38}$ H.-J. Güntherodt and R. Wiesendanger, Theory of Scanning Tunneling Microscopy and related Methods, 1st ed. (Springer Series in Surface Science, Springer, Berlin, 1993). 
39 in Forces in Scanning Probe Methods, Vol. E 286 of NATO ASI, NATO, edited by H.-J. Güntherodt, D. Anselmetti, and E. Meyer (Kluwer, Dordrecht, 1995).

${ }^{40}$ M. Specht, J. D. Pedarnig, W. M. Heckl, and T. W. Hänsch, Phys. Rev. Lett. 68, 476 (1992).

${ }^{41}$ F. Zenhausern, M. P. O’Boyle, and H. K. Wickramasinghe, Appl. Phys Lett. 65, 1623 (1994).

${ }^{42}$ F. Zenhausern, Y. Martin, and H. K. Wickramasinghe, Science 269, 1083 (1995).

${ }^{43}$ U. C. Fischer, U. T. Dürig, and D. W. Pohl, Appl. Phys. Lett. 52, 249 (1988).

${ }^{44}$ J. Koglin, U. C. Fischer, and H. Fuchs, Phys. Rev. B 55, 7977 (1997).

${ }^{45}$ S. Grésillon et al., Phys. Rev. Let. 82, 4520 (1999).

${ }^{46}$ S. Grésillon, H. Cory, J. C. Rivoal, and A. C. Boccara, J. Opt. A: Pure Appl. Opt. 1, 178 (1999).

${ }^{47}$ E. Betzig, P. L. Finn, and S. J. Weiner, Appl. Phys. Lett. 60, 2484 (1992).

${ }^{48}$ U. Fischer and D. Pohl, Phys. Rev. Lett. 62, 458 (1989).

${ }^{49}$ A. Harootunian, E. Betzig, M. Isaacson, and A. Lewis, Appl. Phys. Lett. 49, 674 (1986).

${ }^{50}$ L. Novotny, D. W. Pohl, and P. Regli, Ultramicroscopy 11, 1768 (1995).

${ }^{51}$ L. Novotny, B. Hecht, and D. W. Pohl, J. Appl. Phys. 81, 1798 (1997).

${ }^{52}$ A. Dereux and D. Pohl, in Near-field optics, Vol. E 242 of NATO ASI, NATO, edited by D. Pohl and D. Courjon (Kluwer, Dordrecht, 1993), pp. 189-198.

${ }^{53}$ A. Dereux, J. P. Vigneron, P. Lambin, and A. A. Lucas, Physica B 175, 65 (1991).

${ }^{54}$ C. Girard and X. Bouju, J. Chem. Phys. 95, 2056 (1991).

${ }^{55}$ C. Girard, A. Dereux, and O. J. F. Martin, Phys. Rev. B 49, 13872 (1994). 
${ }^{56}$ P. Morse and H. Feshbach, Methods of theoretical physics (McGraw-Hill, New-York, 1953).

${ }^{57}$ H. Levine and J. Schwinger, Comm. Pure App. Math. 3, 355 (1950).

${ }^{58}$ C. Girard and X. Bouju, J. Opt. Soc. Am. B 9, 298 (1992).

${ }^{59}$ E. Economou, Green's functions in quantum physics, Vol. 7 of Springer Series in SolidState Science, 2nd ed. (Springer, Berlin, 1983).

${ }^{60}$ O. J. F. Martin, C. Girard, D. R. Smith, and S. Schultz, Phys. Rev. Let. 82, 315 (1999).

${ }^{61}$ C. Girard, A. Dereux, and O. J. F. Martin, Surf. Sc. 295, 445 (1993).

${ }^{62}$ C. Girard, A. Dereux, O. J. F. Martin, and M. Devel, Phys. Rev. B 50, 14467 (1994).

${ }^{63}$ O. J. F. Martin, C. Girard, and A. Dereux, Phys. Rev. Lett. 74, 526 (1995).

${ }^{64}$ M. Born and E. Wolf, Principles of optics (Pergamon, Oxford, 1964).

${ }^{65}$ C. Girard and D. Courjon, Phys. Rev. B 42, 9340 (1990).

${ }^{66}$ O. Keller, M. Xiao, and S. Bozhevolnyi, Surf. Sci. 280, 217 (1992).

${ }^{67}$ O. Keller, Physics Reports 268, 85 (1996).

${ }^{68}$ L. D. Landau and E. M. Lifshitz, Field theory, 3rd ed. (Pergamon press, London, 1960).

${ }^{69}$ D. Barchiesi et al., Phys. Rev. E 54, 4285 (1996).

${ }^{70}$ J. Weeber et al., Phys. Rev. Let. 77, 5332 (1996).

${ }^{71}$ J. C. Weeber, Ph.D. thesis, Burgundy University, Dijon, France, 1996.

${ }^{72}$ C. Girard, A. Dereux, O. J. F. Martin, and M. Devel, Phys. Rev. B 52, 2889 (1995).

${ }^{73}$ J. Krenn et al., Phys. Rev. B 60, 5029 (1999).

${ }^{74}$ A. Lewis and K. Lieberman, Nature 354, 214 (1991).

${ }^{75}$ E. Betzig and R. J. Chichester, Science 262, 1422 (1993). 
${ }^{76}$ S. Nie and R. N. Zare, Annu. Rev. Biophys. Biomol. Struct. 26, 567 (1997).

${ }^{77}$ C. Girard, O. J. F. Martin, and A. Dereux, Phys. Rev. Lett. 75, 3098 (1995).

${ }^{78}$ L. Novotny, Appl. Phys. Let. 69, 3806 (1996).

${ }^{79}$ L. Novotny, Ph.D. thesis, Swiss Federal Institute of Technology, 1996.

${ }^{80}$ D. Pastré, P. Grossel, and M. Troyon, Opt. Communication 156, 92 (1998).

${ }^{81}$ G. Parent, D. VanLabeke, and D. Barchiesi, J. of Microscopy 194, 281 (1999).

${ }^{82}$ C. Henkel and V. Sandoghdar, Opt. Communication 158, 250 (1998).

${ }^{83}$ J. F. Ward, Rev. Mod. Phys. 37, 1 (1965).

${ }^{84}$ O. J. F. Martin, A. Dereux, and C. Girard, J. Opt. Soc. Am. A 11, 1073 (1994).

${ }^{85}$ O. J. F. Martin, C. Girard, and A. Dereux, Phys. Rev. Lett. 74, 526 (1995).

${ }^{86}$ J. D. Joannopoulos, R. Meade, and J. Winn, Photonic crystals, 1st ed. (Princeton Press, Princeton NJ, 1995).

${ }^{87}$ J. D. Joannopoulos, P. R. Villeneuve, and S. Fan, Nature 386, 143 (1997).

${ }^{88}$ C. Girard, A. Dereux, and C. Joachim, Phys. Rev. E 59, 6097 (1999).

${ }^{89}$ D. Mullin, M. Spajer, and D. Courjon, J. Appl. Phys. 86, xxx (1999). 


\section{TABLES}

TABLE I. For $s$ polarized incident field, contrast in the near-field zone right above a nanoscopic particle deposited on substrate both made of low index dielectric materials.

\begin{tabular}{l|r|r|r|r}
\hline \hline Field & $\theta_{0}$ & Sign & Predicted & Contrast \\
intensity & dependence & of $(\Xi)$ & contrast & decay law \\
\hline \hline Electric & negligible & negative & dark & $r^{-3}$ \\
\hline Magnetic & weak & positive & bright & $r^{-2}$ \\
\hline \hline
\end{tabular}

TABLE II. Same as table I but for a $p$ polarized incident field.

\begin{tabular}{l|r|r|r|r}
\hline \hline Field & $\begin{array}{c}\theta_{0} \\
\text { dependence }\end{array}$ & $\begin{array}{c}\text { Sign } \\
\text { of }(\Xi)\end{array}$ & $\begin{array}{r}\text { Predicted } \\
\text { contrast }\end{array}$ & $\begin{array}{c}\text { Contrast } \\
\text { decay law }\end{array}$ \\
\hline \hline Electric & weak & positive & bright & $r^{-3}$ \\
\hline Magnetic & strong & negative & dark & $r^{-2}$ \\
\hline \hline
\end{tabular}




\section{FIGURES}

FIG. 1. Schematic drawing of two basic illumination configurations. (A) External reflection; (B) Total internal reflection.

FIG. 2. Variation of the exponential decay versus the angle of incidence. The glass sample (index of refraction $n=1.5$ ) is illuminated in total internal reflection ( $s$-polarized mode).

FIG. 3. Model system of a simple surface corrugation. A tranparent substrate, of optical index $n=1.5$, supports a small dielectric sphere of diameter $D$. The system is illuminated in total internal reflection with an incident angle $\theta_{0}$ and $\mathbf{r}_{s}=(0,0, D / 2)$

FIG. 4. Image of microfabricated dielectric samples recorded by an atomic force microscope. (a) The section of the small glass pads is $100 \mathrm{~nm} \times 100 \mathrm{~nm}$ and their height is $70 \mathrm{~nm}$. (b) The section of the small glass pads is $250 \mathrm{~nm} \times 250 \mathrm{~nm}$ and their height is $100 \mathrm{~nm}$.

FIG. 5. PSTM isointensity image of the sample shown in figure 4 (a) for both incident polarizations $p(\mathrm{a})$ et $s(\mathrm{~b})$. The projection of the incident wavevector on the sample surface is directed along $y$.

FIG. 6. PSTM isointensity image of the sample shown in figure 4 (b) for both incident polarizations $p(\mathrm{a})$ et $s(\mathrm{~b})$. The projection of the incident wavevector on the sample surface was tilted approximately 30 degrees relatively to the $y$ direction (nearly pointing towards the upper right corners of the images)

FIG. 7. For both polarizations $p$ (a) and $s$ (b) and not including any tip, theoretical isointensity distributions of $|\mathbf{E}(\mathbf{r}, \omega)|^{2}$. The computation uses de AFM topography of figure 4 (b) as input. 
FIG. 8. Comparison of a theoretical computation (a) with an experimental PSTM image recorded at constant height (b) of a Au particle $\left(100 \times 100 \times 40 \mathrm{~nm}^{3}\right)$ deposited on a ITO glass substrate. The particle is centered at the origin of the coordinates system in the computation (a) (the surface projection of the model particle corresponds to the white square) while it is slightly translated to the left in the experimental image (b).

FIG. 9. (a) Constant height PSTM image recorded above a chain of Au particles (individual size: $100 \times 100 \times 40 \mathrm{~nm}^{3}$ ) separated of each other by a distance of $100 \mathrm{~nm}$ and deposited on an ITO glass substrate. A comparison with a numerical simulation (b) shows that the bright spots are not on top of the Au particles (the surface projections of the particles correspond to the white squares). The intensity scale of experimental data (a) is normalized to the one of the numerical calculation.

FIG. 10. Schematic drawing illustrating a SNOM geometry in the presence of a single molecule.

FIG. 11. (A) Top view of the model structure. Glass pads (section $90 \times 90 \mathrm{~nm}^{2}$, height $60 \mathrm{~nm}$ ) deposited on a flat glass substrate. At a constant height, $160 \mathrm{~nm}$ above the flat surface and for a wavelength in vacuum equal to $543 \mathrm{~nm}$ : (B) Electromagnetic LDOS; (C) Partial LDOS $\rho_{x x}\left(\mathbf{r}_{m}, \omega\right)$; (D) Partial LDOS $\rho_{z z}\left(\mathbf{r}_{m}, \omega\right)$. 

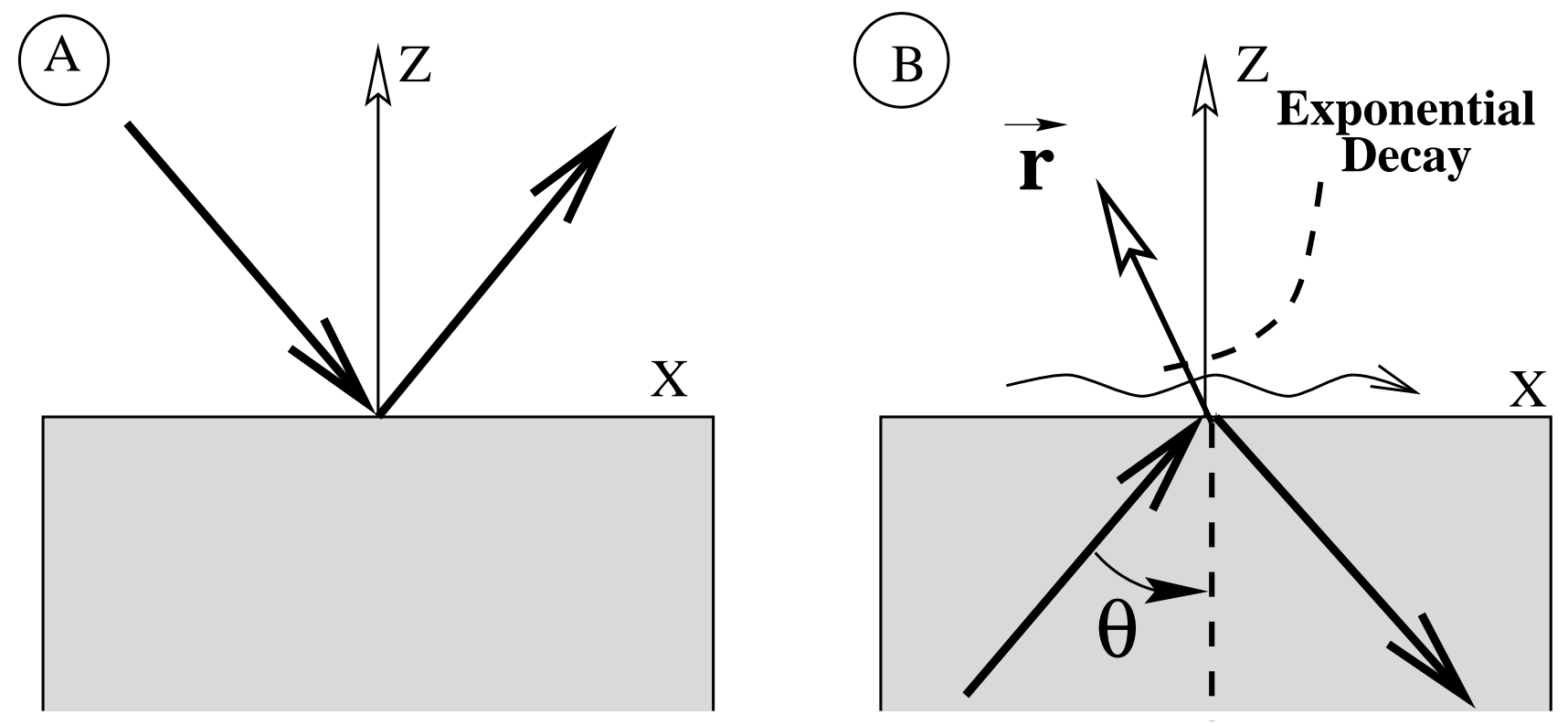

A. Dereux, Ch. Girard, J. C. Weeber: Figure 1 


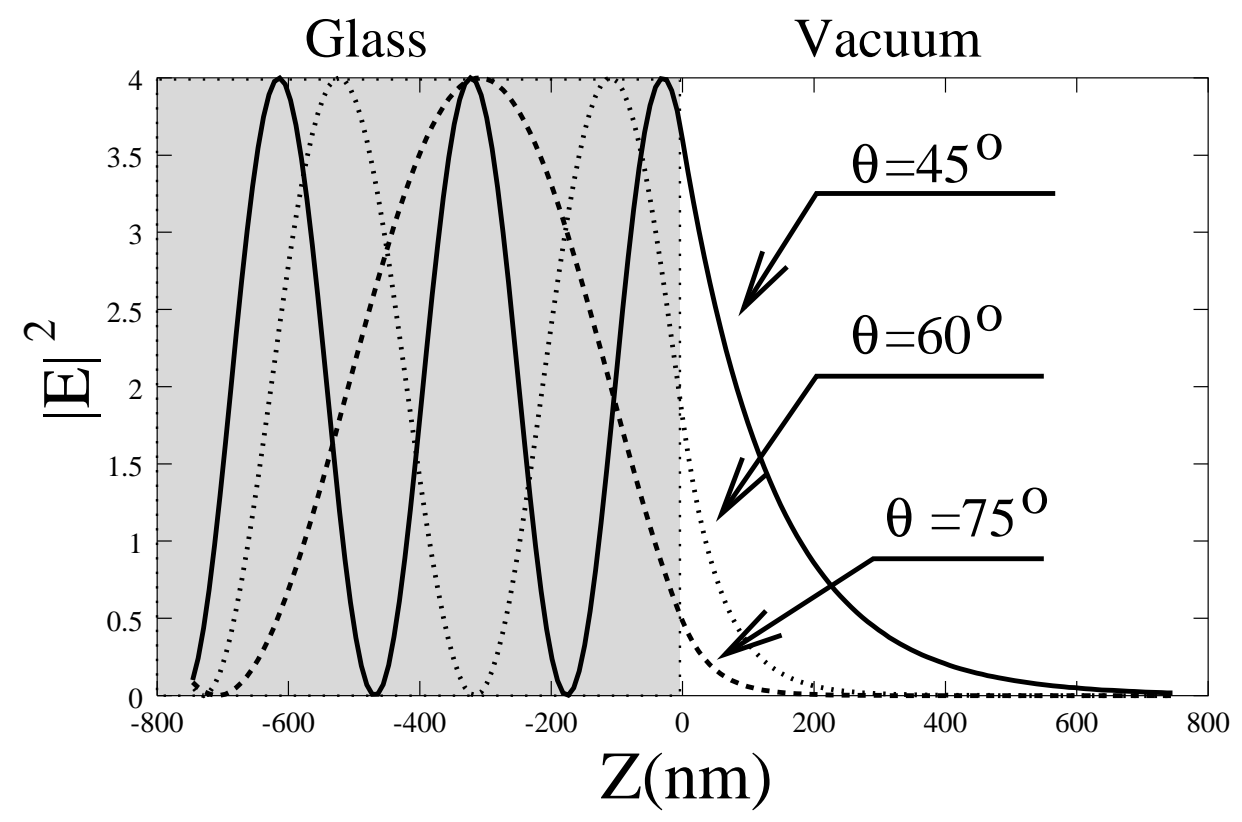

A. Dereux, Ch. Girard, J. C. Weeber: Figure 2 


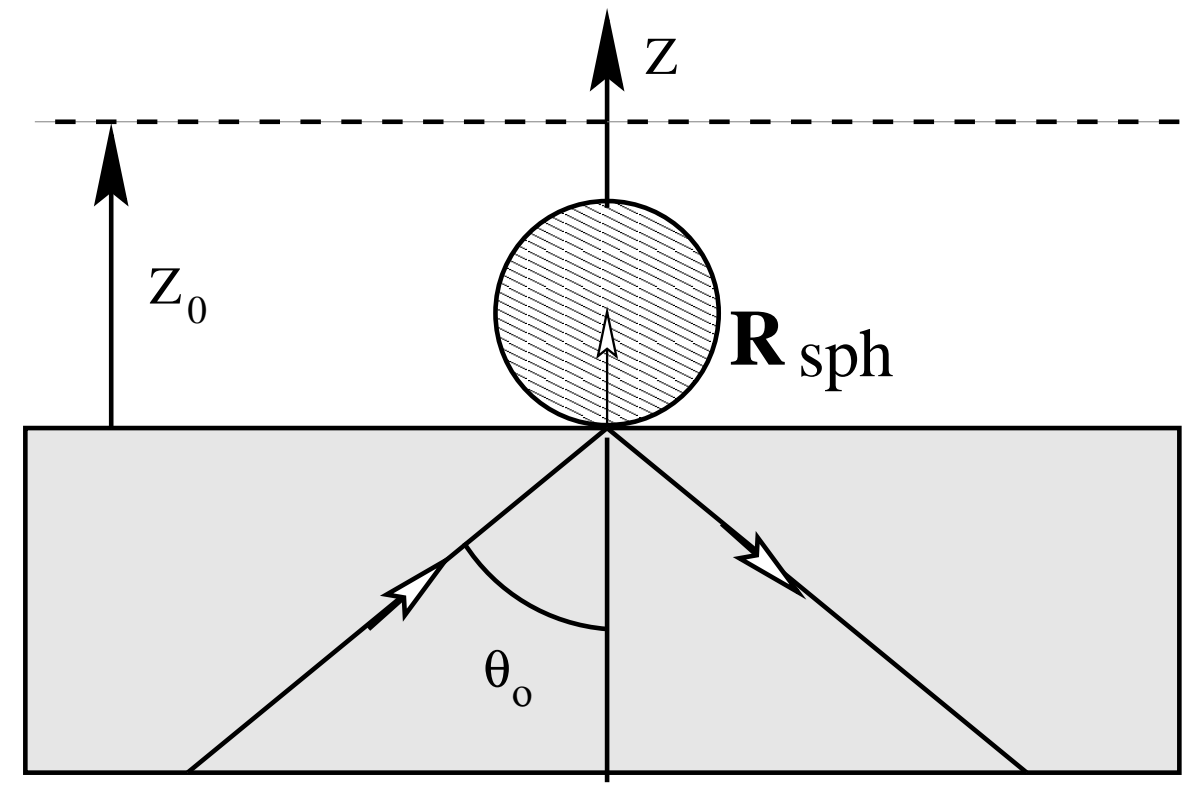

A. Dereux, Ch. Girard, J. C. Weeber: Figure 3 

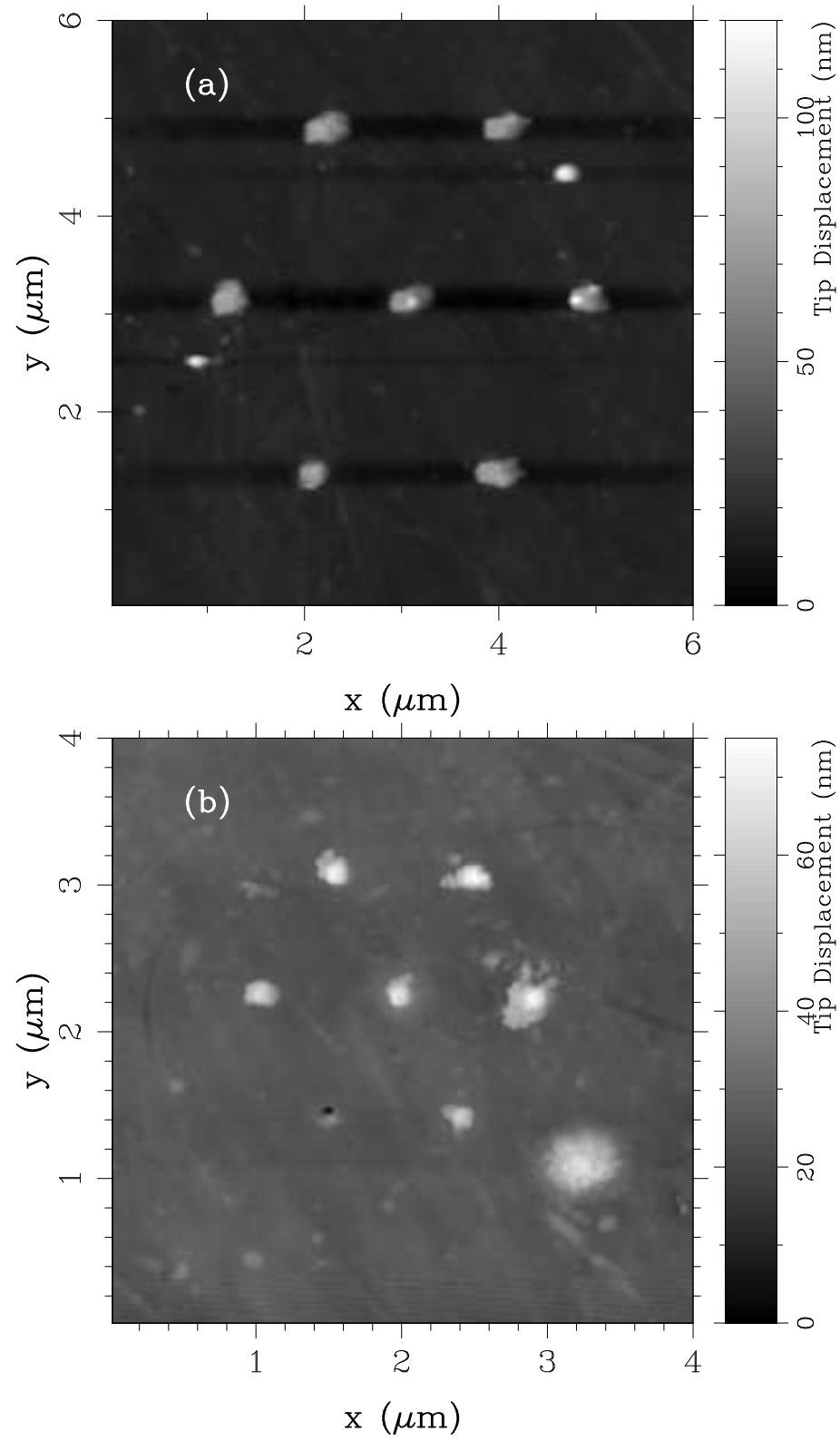

A. Dereux, Ch. Girard, J. C. Weeber: Figure 4 

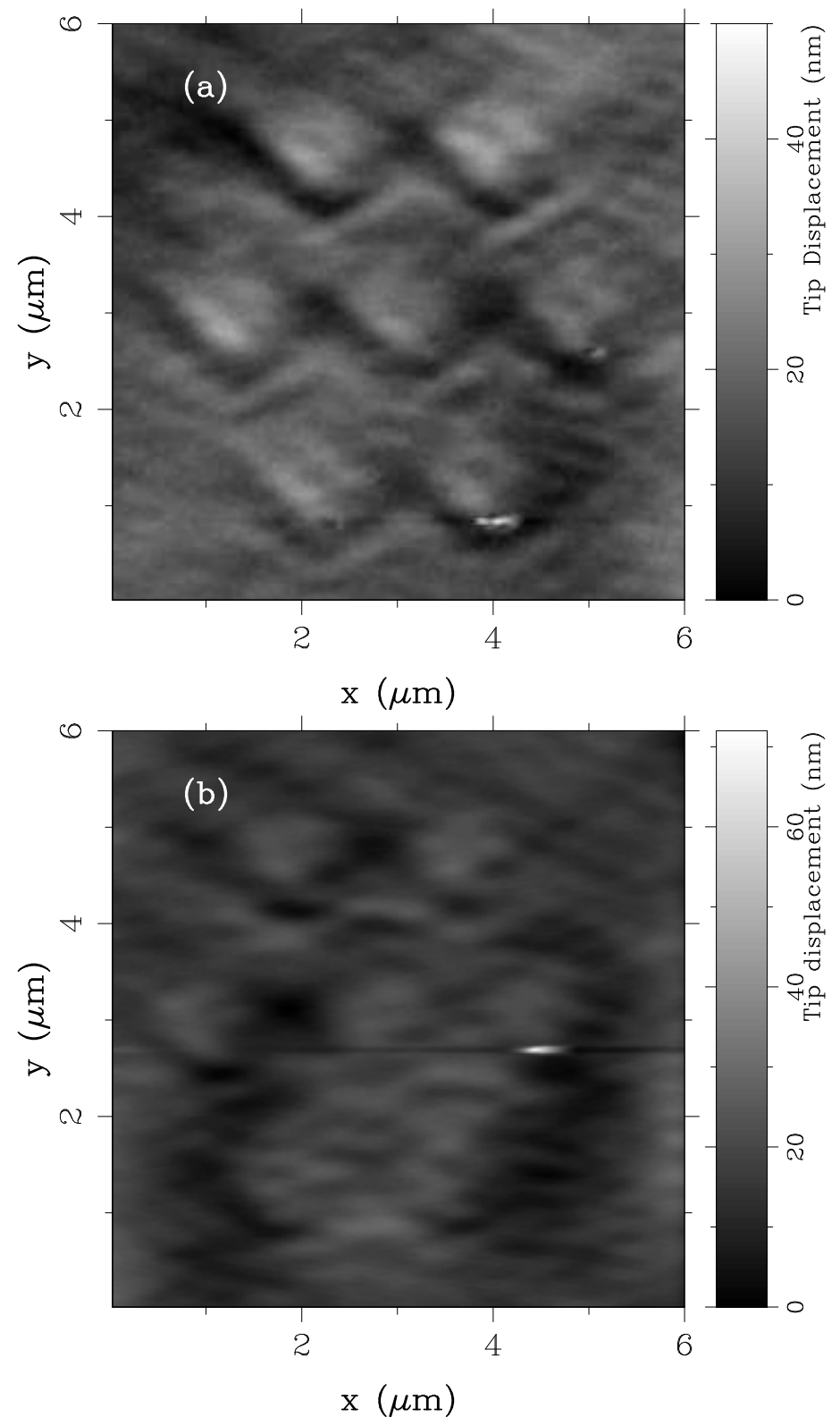

A. Dereux, Ch. Girard, J. C. Weeber: Figure 5 

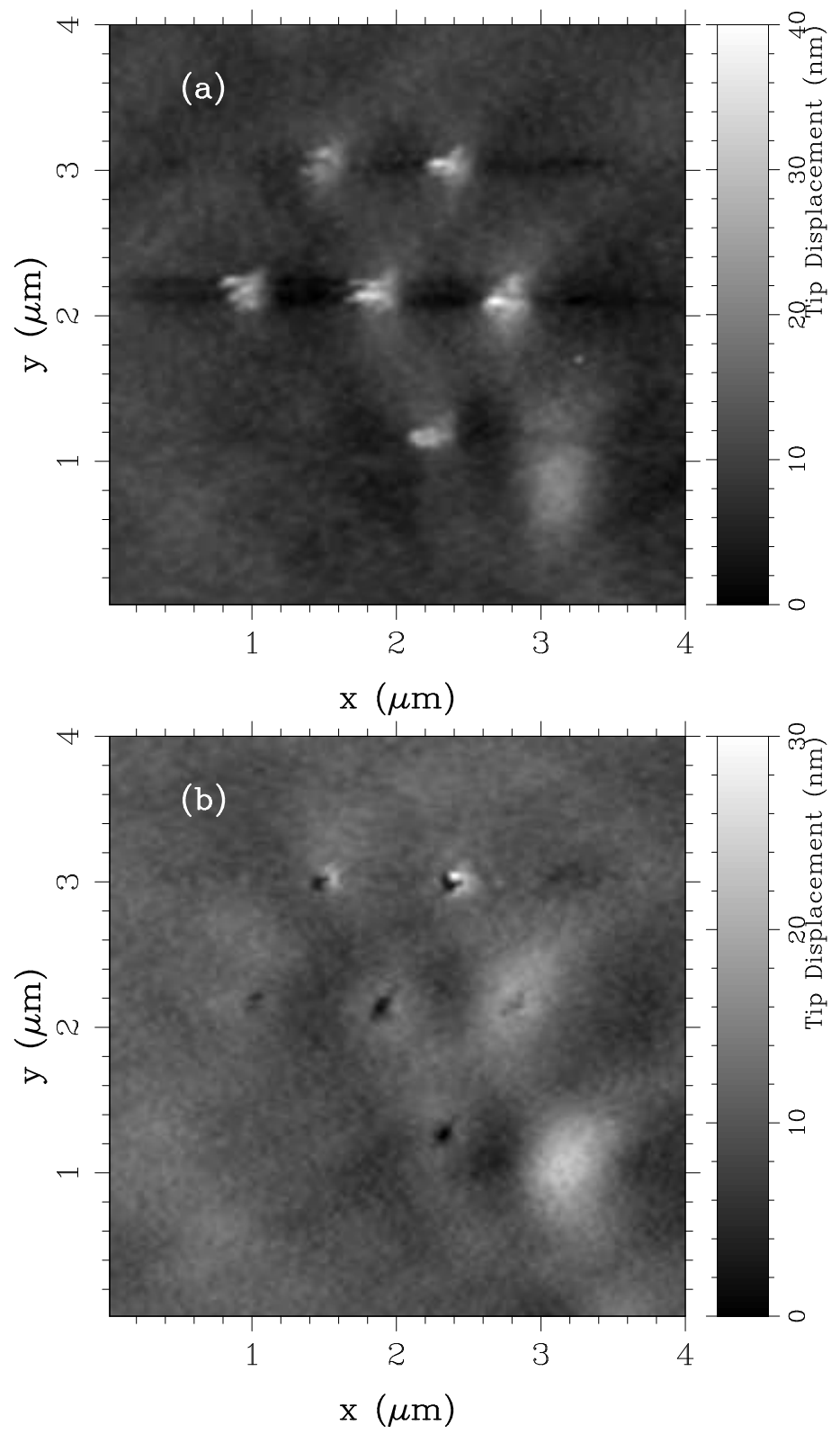

A. Dereux, Ch. Girard, J. C. Weeber: Figure 6 

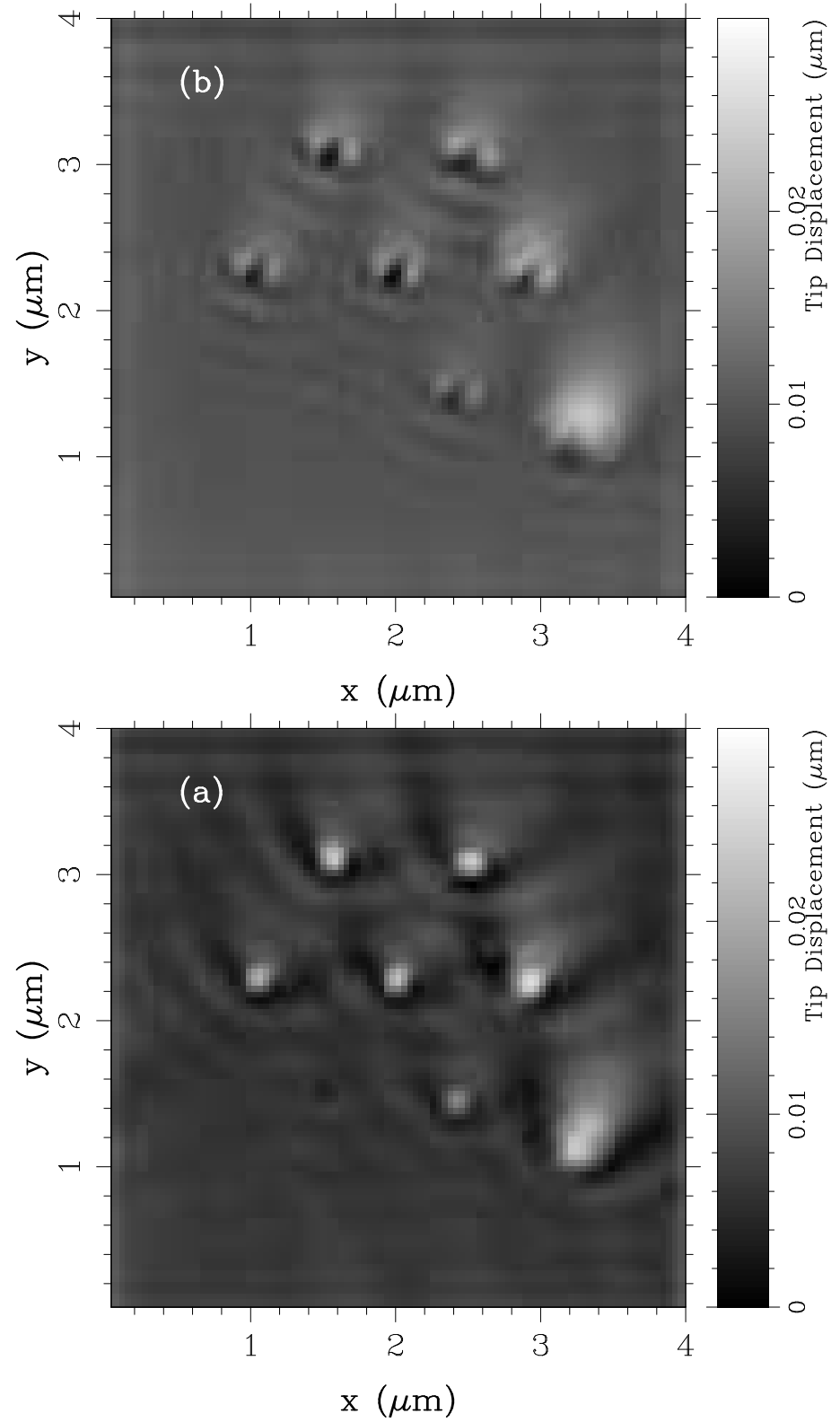

A. Dereux, Ch. Girard, J. C. Weeber: Figure 7 

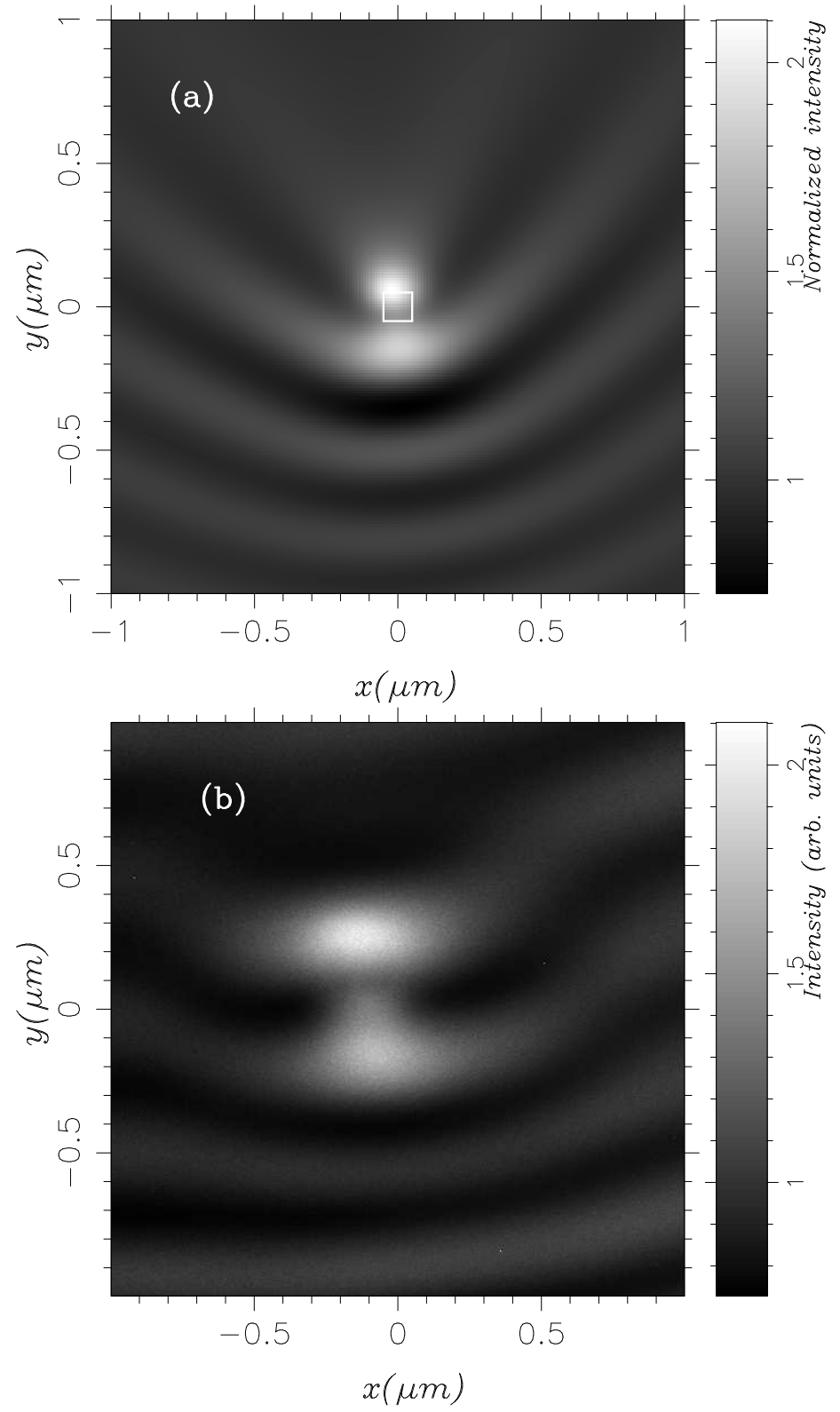

A. Dereux, Ch. Girard, J. C. Weeber: Figure 8 

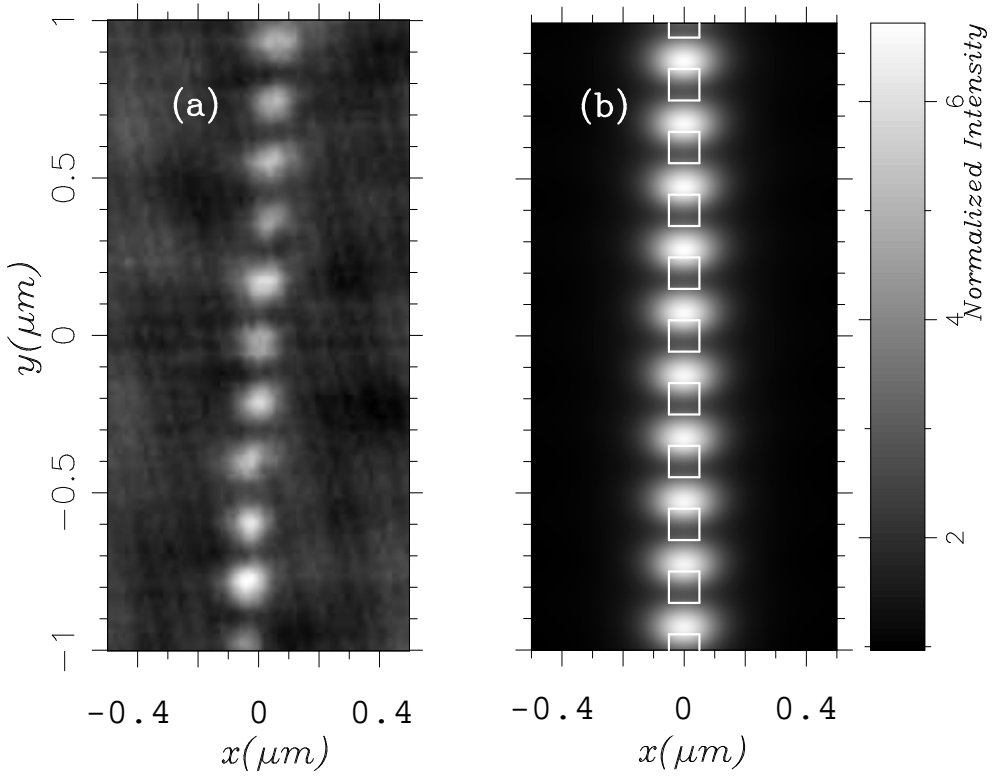

A. Dereux, Ch. Girard, J. C. Weeber: Figure 9 


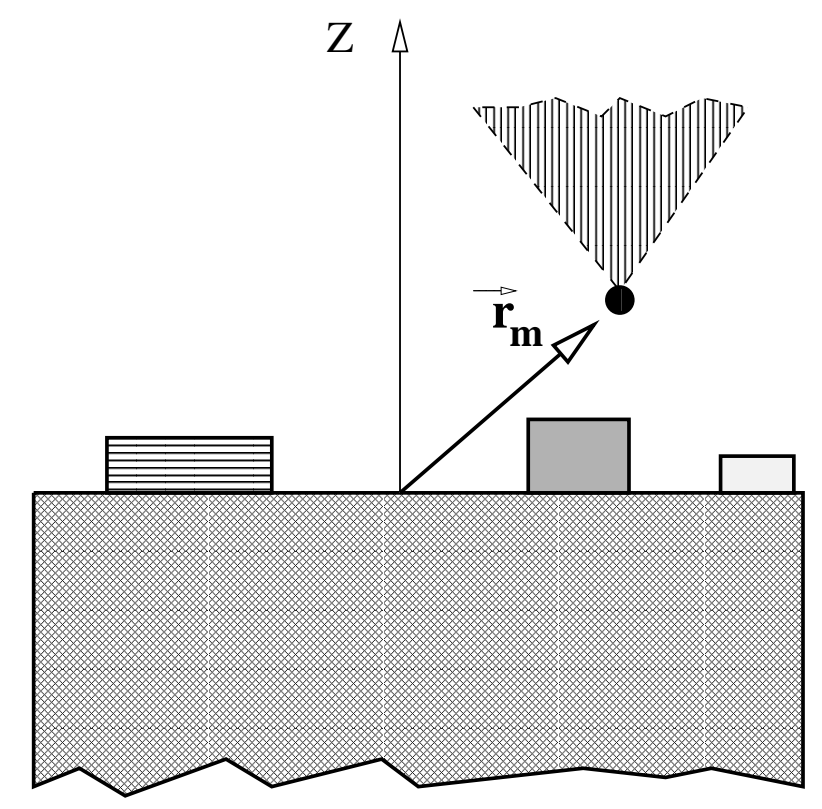

A. Dereux, Ch. Girard, J. C. Weeber: Figure 10 

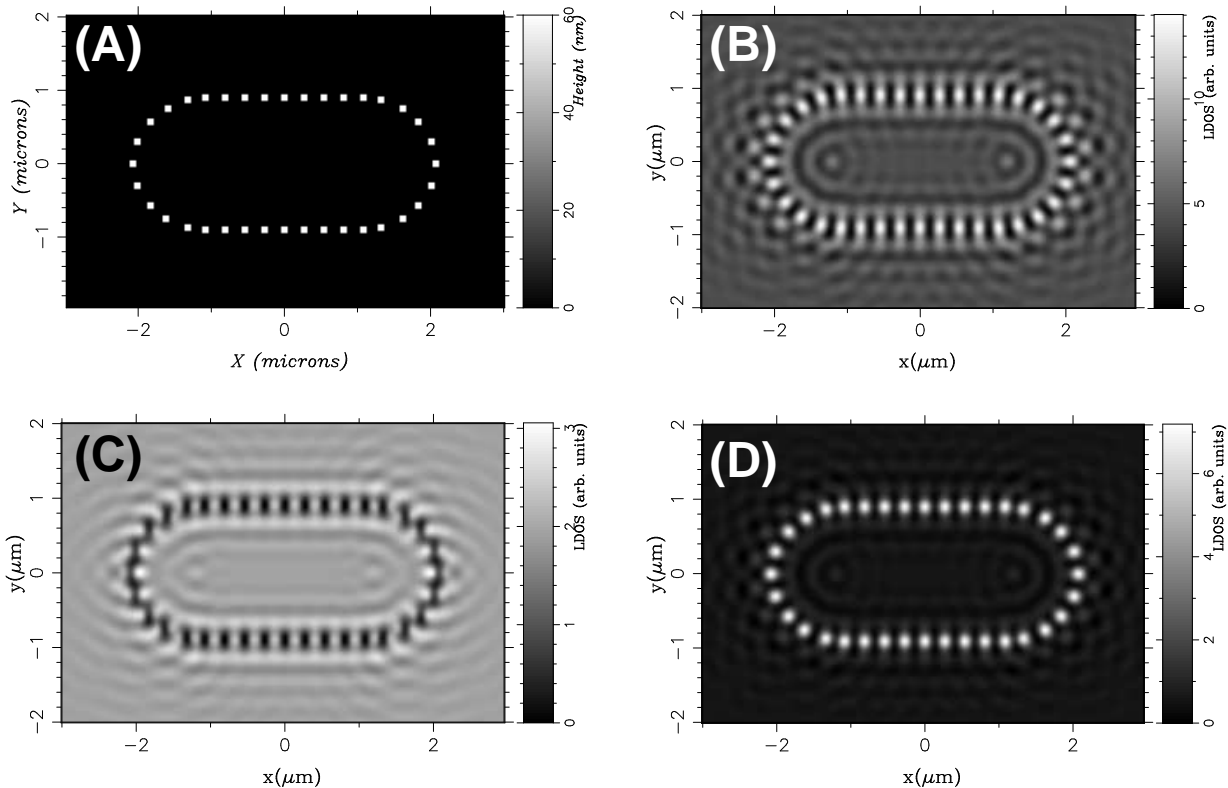

A. Dereux, Ch. Girard, J. C. Weeber: Figure 11 